\title{
Global Dynamics of the Brusselator Equations
}

\author{
Yuncheng You
}

Communicated by Y. Charles Li, received December 29, 2006.

\begin{abstract}
In this work the existence of a global attractor for the solution semiflow of the Brusselator equations is proved. A new decomposition method is introduced to overcome the difficulties in proving the asymptotical compactness of the coupled reaction-diffusion equations whose nonlinearity does not possess dissipative property. It is proved that the Hausdorff dimension and the fractal dimension of the global attractor are finite. The existence of a global attractor with finite dimensionality is also shown by the same approach for the Gray-Scott equations, the Glycolysis equations, and the Schnackenberg equations.
\end{abstract}

\section{Contents}

1. Introduction 167

2. Absorbing Property 172

3. Asymptotical Compactness by Decomposition 176

4. $\kappa$-Contracting Property 181

5. Finite Dimensionality of Global Attractor 190

$\begin{array}{ll}\text { References } & 195\end{array}$

\section{Introduction}

A generic class of nonlinear reaction-diffusion systems is in the form

$$
\begin{aligned}
& \frac{\partial u}{\partial t}=d_{1} \Delta u+a_{1} u+b_{1} v+f(u, v)+g_{1}(x), \\
& \frac{\partial v}{\partial t}=d_{2} \Delta v+a_{2} u+b_{2} v-f(u, v)+g_{2}(x),
\end{aligned}
$$

with homogeneous Dirichlet or Neumann boundary condition on a bounded domain $\Omega \subset \Re^{n}, n \leq 3$, with locally Lipschitz continuous boundary. It is well known that

1991 Mathematics Subject Classification. 37L30, 35B40, 35B41, 35K55, 35K57, 35Q80.

Key words and phrases. Brusselator, Gray-Scott equation, global dynamics, global attractor, absorbing set, asymptotic compactness. 
reaction and diffusion of chemical or biochemical species can produce a variety of spatial patterns. This class of reaction diffusion systems includes some significant pattern formation equations arising from the modeling of kinetics of chemical or biochemical reactions and from the biological pattern formation theory.

In this group, the following four systems are typically important and serve as mathematical models in physical chemistry and in biology:

Brusselator model: $a_{1}=-(b+1), b_{1}=0, a_{2}=b, b_{2}=0, f=u^{2} v, g_{1}=$ $a, g_{2}=0$, where $a$ and $b$ are positive constants.

Gray-Scott model: $a_{1}=-(F+k), b_{1}=0, a_{2}=0, b_{2}=-F, f=u^{2} v, g_{1}=$ $0, g_{2}=F$, where $F$ and $k$ are positive constants.

Glycolysis model: $a_{1}=-1, b_{1}=k, a_{2}=0, b_{2}=-k, f=u^{2} v, g_{1}=\rho, g_{2}=\delta$, where $k, \rho$, and $\delta$ are positive constants.

Schnackenberg model: $a_{1}=-k, b_{1}=a_{2}=b_{2}=0, f=u^{2} v, g_{1}=a, g_{2}=b$, where $k, a$, and $b$ are positive constants.

The Gray-Scott model was originated from describing an isothermal autocatalytic, continuously fed, unstirred reaction and diffusion of two chemicals $\mathrm{U}$ and $\mathrm{V}$ with concentrations $u(t, x)$ and $v(t, x)$, cf. $[\mathbf{1 1}, \mathbf{1 2}]$. Since 1993, a variety of spatial patterns generated by the steady state solutions and longtime evolving solutions have been exposed by experiments $[\mathbf{1 1}, \mathbf{1 5}]$, by numerical simulations $[\mathbf{1 8}]$, or by mathematical analysis $[6,32]$.

The Brusselator is originally a system of two ordinary differential equations as the reaction rate equations for an autocatalytic, oscillating chemical reaction, cf. $[\mathbf{3}, \mathbf{1 9}, \mathbf{2 6}]$. The name is after the hometown of scientists who proposed it. In many autocatalytic systems, even ODEs [26], complex dynamics are seen, including multiple steady states, periodic orbits, and bifurcations.

The Belousov-Zhabotinsky reaction [8] is a generic chemical reaction in which the concentrations of the reactants exhibit somewhat oscillating behavior. In particular, the Brusselator model describes the case in which the chemical reactions follow the scheme

$$
\begin{aligned}
\mathrm{A} & \longrightarrow \mathrm{U}, \\
\mathrm{B}+\mathrm{U} & \longrightarrow \mathrm{V}+\mathrm{D}, \\
2 \mathrm{U}+\mathrm{V} & \longrightarrow 3 \mathrm{U}, \\
\mathrm{U} & \longrightarrow \mathrm{E},
\end{aligned}
$$

where $\mathrm{A}, \mathrm{B}, \mathrm{D}, \mathrm{E}, \mathrm{U}$, and $\mathrm{V}$ are chemical compounds. Let $u(t, x)$ and $v(t, x)$ be the concentrations of $\mathrm{U}$ and $\mathrm{V}$, and assume that the concentrations of the input compounds $\mathrm{A}$ and $\mathrm{B}$ are held constant during the reaction process, denoted by $a$ and $b$ respectively. Then one obtains the following system of two nonlinearly coupled reaction-diffusion equations,

$$
\begin{gathered}
\frac{\partial u}{\partial t}=d_{1} \Delta u+u^{2} v-(b+1) u+a, \quad(t, x) \in(0, \infty) \times \Omega, \\
\frac{\partial v}{\partial t}=d_{2} \Delta v-u^{2} v+b u, \quad(t, x) \in(0, \infty) \times \Omega, \\
u(t, x)=v(t, x)=0, \quad t>0, x \in \partial \Omega, \\
u(0, x)=u_{0}(x), v(0, x)=v_{0}(x), \quad x \in \Omega,
\end{gathered}
$$


where $d_{1}, d_{2}, a$, and $b$ are positive constants. Here it is assumed that the rate coefficients for the intermediate reactions are equal to one. Actually the results of this paper will not be affected by taking different reaction coefficients. The system of equations (1.1) and (1.2) is called the Brusselator equations.

Note that there are several known examples of autocatalysis which can be modeled by the Brusselator equations, such as ferrocyanide-iodate-sulphite reaction, chlorite-iodide-malonic acid reaction, arsenite-iodate reaction, some enzyme catalytic reactions, and fungal mycelia growth, cf. $[\mathbf{2}, \mathbf{3}, \mathbf{5}, \mathbf{7}]$.

Since 1970's there have been some but limited studies on the spatial pattern solutions as steady states, their local stabilities and bifurcations for the Brusselator equations. In $[\mathbf{9}, \mathbf{1 6}, \mathbf{2 0}, \mathbf{2 2}, \mathbf{3 3}]$, some Turing patterns generated by the Brusselator equations were studied numerically or analytically, including spike patterns, stripe patterns, and oscillating instabilities. In [1] numerical results were presented, showing mazelike patterns, frustrated hexagonal patterns, and chaotic-looking patterns, for the 2D Brusselator equations and the hyperbolic version with the diffusion flux equations. In [14], under the assumptions of slow input and slow diffusion rates, the existence of a mesa-type patterns for the 1D Brusselator is shown along with a threshold for the local stabilities and a Hopf bifurcation to a breather-type instability by using the singular perturbation method.

For the Brusselator equations and the other three model equations of space dimension $n \leq 3$, however, we have not seen substantial research results in the front of global dynamics. In this paper, without any restrictive assumptions, we show the existence of a global attractor in the $L^{2}$ phase space for the solution semiflow of the Brusselator equations (1.1) and (1.2) with homogeneous Dirichlet boundary conditions.

The basic theory of global attractors and applications can be found in $[\mathbf{1 3}, \mathbf{2 4}$, 29] and many references therein. Since the years 1980 the existence of a global attractor has been proved for quite a few dissipative parabolic equations and damped nonlinear wave equations. The typical dissipativity for a single reaction-diffusion equation is embodied in the asymptotic sign condition on the right-hand side nonlinear function $f(u)$, i.e.

$$
\limsup _{|s| \rightarrow \infty} \frac{f(s)}{s} \leq 0
$$

As for systems of two or more reaction diffusion equations, the corresponding asymptotic sign condition in vector version is usually not satisfied. A limited results on the existence of a global attractor were proved for partially dissipative reactiondiffusion systems, such as FitzHugh-Nagumo equations. Some results based on the construction of a positively invariant region in $\Re^{n}$ in general provide local attractors only.

For the Brusselator equations (1.1) and (1.2), the essential difficulties in proving the existence of a global attractor lie in the fact that the oppositely interactive polynomial nonlinearity in the two coupled equations does not possess partial dissipativity or asymptotic dissipativity, which causes certain obstacle in proving the existence of absorbing sets and even more challenge in showing the asymptotical compactness. In this paper, a new decomposition technique is explored and used to show the $\kappa$-contraction of the solution semiflow. 
We start with the formulation of an evolutionary equation associated with the Brusselator equations. We shall introduce certain concepts and present the main result.

Define the product Hilbert spaces as follows,

$$
\begin{aligned}
H & =L^{2}(\Omega) \times L^{2}(\Omega), \\
E & =H_{0}^{1}(\Omega) \times H_{0}^{1}(\Omega), \\
W & =\left(H_{0}^{1}(\Omega) \cap H^{2}(\Omega)\right) \times\left(H_{0}^{1}(\Omega) \cap H^{2}(\Omega)\right) .
\end{aligned}
$$

The norm and inner-product of $H$ or $L^{2}(\Omega)$ will be denoted by $\|\cdot\|$ and $\langle\cdot, \cdot\rangle$, respectively. The norm of $L^{p}(\Omega)$ will be denoted by $\|\cdot\|_{L^{p}}$. By the Poincaré inequality with the homogeneous Dirichlet boundary condition as specified, there is a constant $\gamma>0$ such that

$$
\|\nabla \varphi\|^{2} \geq \gamma\|\varphi\|^{2}, \quad \text { for } \varphi \in E \text { or } H_{0}^{1}(\Omega),
$$

and we shall take $\|\nabla \varphi\|$ for the equivalent norm of the space $E$ and of the space $H_{0}^{1}(\Omega)$. We use $|\cdot|$ to denote an absolute value or a vector norm in a Euclidean space.

It is easy to check that, by the Lumer-Phillips theorem and the analytic semigroup generation theorem [24], the densely defined, sectorial operator

$$
A=\left(\begin{array}{cc}
d_{1} \Delta & 0 \\
0 & d_{2} \Delta
\end{array}\right): D(A)(=W) \longrightarrow H
$$

is the generator of an analytic $C_{0}$-semigroup on the Hilbert space $H$. By the fact that $H_{0}^{1}(\Omega) \hookrightarrow L^{6}(\Omega)$ is a continuous embedding for $n \leq 3$ and using the generalized Hölder inequality,

$$
\left\|u^{2} v\right\| \leq\|u\|_{L^{6}}^{2}\|v\|_{L^{6}}, \quad \text { for } u, v \in L^{6}(\Omega),
$$

one can verify that the nonlinear mapping

$$
F(u, v)=\left(\begin{array}{c}
u^{2} v-(b+1) u+a \\
-u^{2} v+b u
\end{array}\right): E \longrightarrow H
$$

is well defined on $E$ and locally Lipschitz continuous. Then by the theory of evolutionary equations [24], one can easily show the local existence and uniqueness of the strong solution

$$
u \in C\left(\left[0, T_{\max }\right) ; H\right) \cap L^{2}\left(0, T_{\max } ; E\right),
$$

where $\left[0, T_{\max }\right)$ is the maximal interval of existence, of the Brusselator evolution equation

$$
\frac{d w}{d t}=A w+F(w), \quad t>0,
$$

where $w(t)=\operatorname{col}(u(t, \cdot), v(t, \cdot))$, or written as $(u(t, \cdot), v(t, \cdot))$, for any initial data $w(0)=w_{0}=\left(u_{0}, v_{0}\right) \in H$.

We refer to $[\mathbf{2 4}]$ and $[\mathbf{2 9}]$ for the concepts and basic facts in the theory of infinite dimensional dynamical systems, including few given below for clarity. 
Definition 1. Let $M$ be a complete metric space. A time-dependent family of maps $\{S(t)\}_{t \geq 0}$ is called a semiflow (or semigroup) on $M$, if the following conditions are satisfied:

(i) $S(0) w=w$, for all $w \in M$. Namely, $S(0)=I$, the identity map on $M$.

(ii) $S(t+s)=S(t) S(s)$ for all $t, s \geq 0$.

(iii) The map: $(t, w) \mapsto S(t) w$ is continuous from $[0, \infty) \times M$ into $M$.

Definition 2. Let $\{S(t)\}_{t \geq 0}$ be a semiflow on a complete metric space $M$. A subset $B_{0}$ of $M$ is called an absorbing set in $M$ if, for any bounded subset $B \subset M$, there is some finite time $t_{0} \geq 0$ depending on $B$ such that $S(t) B \subset B_{0}$ for all $t \geq t_{0}$.

Definition 3. Let $\{S(t)\}_{t \geq 0}$ be a semiflow on a complete metric space $M$ whose metric is denoted by $d(\cdot, \cdot)$. A subset $\mathcal{A}$ of $M$ is called a global attractor for this semiflow, if $\mathcal{A}$ has the following properties:

(i) $\mathcal{A}$ is a nonempty, compact, and invariant set in the sense that $S(t) \mathcal{A}=\mathcal{A}$ for any $t \geq 0$.

(ii) $\mathcal{A}$ attracts any bounded set $B$ of $M$ in the sense that, in terms of the Hausdorff distance,

$$
\operatorname{dist}(S(t) B, \mathcal{A})=\sup _{x \in B} \inf _{y \in \mathcal{A}} d(x, y) \rightarrow 0, \quad \text { as } t \rightarrow \infty .
$$

Definition 4. A semiflow $\{S(t)\}_{t \geq 0}$ on a complete metric space $M$ is called asymptotically compact if for any sequences $\left\{u_{n}\right\}$ which is bounded in $M$ and $\left\{t_{n}\right\} \subset(0, \infty)$ with $t_{n} \rightarrow \infty$, there exist subsequences $\left\{u_{n_{k}}\right\}$ of $\left\{u_{n}\right\}$ and $\left\{t_{n_{k}}\right\}$ of $\left\{t_{n}\right\}$, such that $\lim _{k \rightarrow \infty} S\left(t_{n_{k}}\right) w_{n_{k}}$ exists in $M$.

Here is the main result of this paper.

Theorem 1 (Main Theorem). For any positive parameters $d_{1}, d_{2}$, $a$, and $b$, there exists a global attractor $\mathcal{A}$ in $H$ for the solution semiflow $\{S(t)\}_{t \geq 0}$ generated by the Brusselator equations (1.1)-(1.2).

By the same approach the existence of a global attractor for the Gray-Scott equations, the Glycolysis equations, and the Schnackenberg equations $[\mathbf{2 3}, \mathbf{3 1}]$ in space dimension $n \leq 3$ is also proved.

In $[\mathbf{2 4}$, Chapter 2], the basic existence theory of global attractors is provided, which can be concisely stated in the following lemma.

Lemma 1. Let $\{S(t)\}_{t \geq 0}$ be a semiflow on a Banach space $X$, which has the following two properties:

(i) there exists a bounded absorbing set $B_{0} \subset X$ for $\{S(t)\}_{t \geq 0}$, and

(ii) $\{S(t)\}_{t \geq 0}$ is asymptotically compact on $X$.

Then there exists a global attractor $\mathcal{A}$ for $\{S(t)\}_{t \geq 0}$, which is the $\omega$-limit set of $B_{0}$,

$$
\mathcal{A}=\omega\left(B_{0}\right) \stackrel{\text { def }}{=} \bigcap_{\tau \geq 0} C l_{X} \bigcup_{t \geq \tau}\left(S(t) B_{0}\right) .
$$

In Section 2 we shall prove the absorbing property of the Brusselator semiflow. A new decomposition approach is presented in Section 3, which is used to prove the $\kappa$-contracting property in Section 4 . Thus the existence of a global attractor is proved for the Brusselator equations and for the other three model equations. In Section 5 we prove that the global attractors for all these equations have finite Hausdorff dimensions and finite fractal dimensions respectively. 


\section{Absorbing Property}

In the sequal, we shall write $u(t, x)$ and $v(t, x)$ simply as $u(t)$ and $v(t)$, or even as $u$ and $v$, and similarly for other functions of $(t, x)$. Taking the inner product $\langle(1.2), v(t)\rangle$, we get

$$
\begin{aligned}
& \frac{1}{2} \frac{d}{d t}\|v\|^{2}+d_{2}\|\nabla v\|^{2}=\int_{\Omega}\left(-u^{2} v^{2}+b u v\right) d x \\
& =\int_{\Omega}-\left(u v-\frac{b}{2}\right)^{2} d x+\frac{1}{4} b^{2}|\Omega| \leq \frac{1}{4} b^{2}|\Omega| .
\end{aligned}
$$

It follows that

$$
\frac{d}{d t}\|v\|^{2}+2 \gamma d_{2}\|v\|^{2} \leq b^{2}|\Omega|
$$

which yields

$$
\|v(t)\|^{2} \leq e^{-2 \gamma d_{2} t}\left\|v_{0}\right\|^{2}+\frac{b^{2}|\Omega|}{2 \gamma d_{2}}, \quad \text { for } t \in\left[0, T_{\max }\right) .
$$

Hence, we find that

$$
\limsup _{t \rightarrow \infty}\|v(t)\|^{2} \leq \rho_{0}=\frac{b^{2}|\Omega|}{\gamma d_{2}} .
$$

Moreover, for any $t \geq 0,(2.1)$ also implies that

$$
\begin{aligned}
\int_{t}^{t+1}\|\nabla v(s)\|^{2} d s & \leq \frac{1}{d_{2}}\left(\|v(t)\|^{2}+b^{2}|\Omega|\right) \\
& \leq \frac{1}{d_{2}}\left(e^{-2 \gamma d_{2} t}\left\|v_{0}\right\|^{2}+\frac{b^{2}|\Omega|}{2 \gamma d_{2}}\right)+\frac{b^{2}|\Omega|}{d_{2}} .
\end{aligned}
$$

In order to treat the $u$-component, we add up (1.1) and (1.2) to get an equation for the $\operatorname{sum} z(t)=u(t)+v(t)$, which is

$$
z_{t}=d_{1} \Delta z-z+\left[\left(d_{2}-d_{1}\right) \Delta v+v+a\right] .
$$

Taking the inner-product $\langle(2.5), z(t)\rangle$ we obtain

$$
\begin{aligned}
\frac{1}{2} \frac{d}{d t}\|z\|^{2} & +d_{1}\|\nabla z\|^{2}+\|z\|^{2}=\int_{\Omega}\left[\left(d_{2}-d_{1}\right) \Delta v+v+a\right] z d x \\
& =\left(d_{1}-d_{2}\right)\langle\nabla v, \nabla z\rangle+\langle v, z\rangle+\langle a, z\rangle \\
& \leq\left|d_{1}-d_{2}\right|\|\nabla v\|\|\nabla z\|+\|v\|\|z\|+a|\Omega|^{1 / 2}\|z\| \\
& \leq \frac{d_{1}}{2}\|\nabla z\|^{2}+\frac{\left|d_{1}-d_{2}\right|^{2}}{2 d_{1}}\|\nabla v\|^{2}+\frac{1}{4}\|z\|^{2}+\|v\|^{2}+\frac{1}{4}\|z\|^{2}+a^{2}|\Omega|,
\end{aligned}
$$

which implies that 


$$
\begin{aligned}
\frac{d}{d t}\|z\|^{2} & +d_{1}\|\nabla z\|^{2}+\|z\|^{2} \leq \frac{\left|d_{1}-d_{2}\right|^{2}}{d_{1}}\|\nabla v\|^{2}+2\|v\|^{2}+2 a^{2}|\Omega| \\
& \leq \frac{\left|d_{1}-d_{2}\right|^{2}}{d_{1}}\|\nabla v\|^{2}+2 e^{-2 \gamma d_{2} t}\left\|v_{0}\right\|^{2}+\frac{b^{2}|\Omega|}{\gamma d_{2}}+2 a^{2}|\Omega| .
\end{aligned}
$$

Let

$$
C\left(v_{0}, t\right)=2 e^{-2 \gamma d_{2} t}\left\|v_{0}\right\|^{2}+\left(\frac{b^{2}}{\gamma d_{2}}+2 a^{2}\right)|\Omega|
$$

Then we get

$$
\frac{d}{d t}\|z\|^{2}+d_{1}\|\nabla z\|^{2}+\|z\|^{2} \leq \frac{\left|d_{1}-d_{2}\right|^{2}}{d_{1}}\|\nabla v\|^{2}+C\left(v_{0}, t\right)
$$

Integration of inequality (2.6) shows that the strong solution $z(t)$ of equation (2.5) satisfies the estimate

$$
\begin{aligned}
\|z(t)\|^{2} & \leq\left\|u_{0}+v_{0}\right\|^{2}+\frac{\left|d_{1}-d_{2}\right|^{2}}{d_{1}} \int_{0}^{t}\|\nabla v(s)\|^{2} d s \\
& +\frac{1}{\gamma d_{2}}\left\|v_{0}\right\|^{2}+t\left(\frac{b^{2}}{\gamma d_{2}}+2 a^{2}\right)|\Omega| .
\end{aligned}
$$

From (2.1) we see that

$$
d_{2} \int_{0}^{t}\|\nabla v(s)\|^{2} d s \leq\left\|v_{0}\right\|^{2}+b^{2}|\Omega| t
$$

so that, for $t \in\left[0, T_{\max }\right)$,

$$
\begin{aligned}
\|z(t)\|^{2} & \leq\left\|u_{0}+v_{0}\right\|^{2}+\frac{\left|d_{1}-d_{2}\right|^{2}}{d_{1} d_{2}}\left(\left\|v_{0}\right\|^{2}+b^{2}|\Omega| t\right) \\
& +\frac{1}{\gamma d_{2}}\left\|v_{0}\right\|^{2}+\left(\frac{b^{2}}{\gamma d_{2}}+2 a^{2}\right)|\Omega| t \\
& \leq\left\|u_{0}+v_{0}\right\|^{2}+\left(\frac{1}{\gamma d_{2}}+\frac{\left|d_{1}-d_{2}\right|^{2}}{d_{1} d_{2}}\right)\left\|v_{0}\right\|^{2} \\
& +\left(\left(\frac{1}{\gamma d_{2}}+\frac{\left|d_{1}-d_{2}\right|^{2}}{d_{1} d_{2}}\right) b^{2}+2 a^{2}\right)|\Omega| t .
\end{aligned}
$$

Therefore, we have shown the global existence of the strong solutions of the Brusselator evolutionary equation (1.6), as stated in the following lemma.

Lemma 2. For any initial data $w_{0}=\left(u_{0}, v_{0}\right) \in H$, there exists a unique, global, strong solution $w(t)=(u(t), v(t)), t \in[0, \infty)$, of the Brusselator evolutionary equation (1.6). 
Proof. Note that

$$
\|u(t)\| \leq\|z(t)\|+\|v(t)\| \quad \text { and } \quad\|w(t)\|^{2}=\|u(t)\|^{2}+\|v(t)\|^{2} .
$$

The proof is done by (2.2) and (2.8), which means that the strong solution $w(t)$ of the equation (1.6) will never blow up in $H$ at any finite time.

Due to Lemma 2, the family of global strong solutions $\left\{w\left(t ; w_{0}\right), t \geq 0, w_{0} \in H\right\}$ defines a semiflow on $H$,

$$
S(t): w_{0} \mapsto w\left(t ; w_{0}\right), \quad w_{0} \in H, t \geq 0,
$$

which is called the Brusselator semiflow, generated by the Brusselator equations.

Lemma 3. There exists a bounded absorbing set $B_{0}$ in $H$ for the Brusselator semiflow $\{S(t)\}_{t \geq 0}$,

$$
B_{0}=\left\{\|w\| \in H:\|w\|^{2} \leq K_{0}\right\},
$$

where $K_{0}$ is a positive constant independent of any initial data.

Proof. From (2.6) we can deduce that

$$
\frac{d}{d t}\left(e^{t}\|z(t)\|^{2}\right) \leq \frac{\left|d_{1}-d_{2}\right|^{2}}{d_{1}} e^{t}\|\nabla v(t)\|^{2}+C\left(v_{0}, t\right) e^{t} .
$$

Integrate (2.9) to obtain

$$
\|z(t)\|^{2} \leq e^{-t}\left\|u_{0}+v_{0}\right\|^{2}+\frac{\left|d_{1}-d_{2}\right|^{2}}{d_{1}} \int_{0}^{t} e^{-(t-\tau)}\|\nabla v(\tau)\|^{2} d \tau+C_{1}\left(v_{0}, t\right)
$$

where

$$
\begin{aligned}
C_{1}\left(v_{0}, t\right) & =2 e^{-t} \int_{0}^{t} e^{\left(1-2 \gamma d_{2}\right) \tau} d \tau\left\|v_{0}\right\|^{2}+\left(\frac{b^{2}}{\gamma d_{2}}+2 a^{2}\right)|\Omega| . \\
& \leq \alpha(t)\left\|v_{0}\right\|^{2}+\left(\frac{b^{2}}{\gamma d_{2}}+2 a^{2}\right)|\Omega|
\end{aligned}
$$

in which

$$
\alpha(t)= \begin{cases}\frac{2}{\mid 1-2 \gamma d_{2}} e^{-2 \gamma d_{2} t}, & \text { if } 1-2 \gamma d_{2}>0 \\ 2 t e^{-t} \leq 4 e^{-1} e^{-t / 2}, & \text { if } 1-2 \gamma d_{2}=0 \\ \frac{2}{\left|1-2 \gamma d_{2}\right|} e^{-t}, & \text { if } 1-2 \gamma d_{2}<0\end{cases}
$$

We now treat the term

$$
\int_{0}^{t} e^{-(t-\tau)}\|\nabla v(\tau)\|^{2} d \tau
$$

Multiplying (2.1) by $e^{t}$ and then integrating it, we get

$$
\frac{1}{2} \int_{0}^{t}\left(e^{\tau} \frac{d}{d \tau}\|v(\tau)\|^{2}\right) d \tau+d_{2} \int_{0}^{t} e^{\tau}\|\nabla v(\tau)\|^{2} d \tau \leq b^{2}|\Omega| e^{t},
$$

so that, by integration by parts and using (2.2), we have 


$$
\begin{aligned}
& d_{2} \int_{0}^{t} e^{\tau}\|\nabla v(\tau)\|^{2} d \tau \leq b^{2}|\Omega| e^{t}-\frac{1}{2} \int_{0}^{t}\left(e^{\tau} \frac{d}{d \tau}\|v(\tau)\|^{2}\right) d \tau \\
& \quad=b^{2}|\Omega| e^{t}-\frac{1}{2}\left[e^{t}\|v(t)\|^{2}-\left\|v_{0}\right\|^{2}-\int_{0}^{t} e^{\tau}\|v(\tau)\|^{2} d \tau\right] \\
& \quad \leq b^{2}|\Omega| e^{t}+\left\|v_{0}\right\|^{2}+\int_{0}^{t} e^{\tau}\|v(\tau)\|^{2} d \tau \\
& \quad \leq b^{2}|\Omega| e^{t}+\left\|v_{0}\right\|^{2}+\int_{0}^{t} e^{\left(1-2 \gamma d_{2}\right) \tau}\left\|v_{0}\right\|^{2} d \tau+\frac{b^{2}|\Omega|}{2 \gamma d_{2}} e^{t} \\
& \quad \leq b^{2}|\Omega| e^{t}+\left(1+\alpha(t) e^{t}\right)\left\|v_{0}\right\|^{2}+\frac{b^{2}|\Omega|}{2 \gamma d_{2}} e^{t} \\
& \quad=\left(1+\frac{1}{2 \gamma d_{2}}\right) b^{2}|\Omega| e^{t}+\left(1+\alpha(t) e^{t}\right)\left\|v_{0}\right\|^{2}, \quad \text { for any } t \geq 0 .
\end{aligned}
$$

Substituting (2.13) into (2.10) and using (2.11), we obtain that, for all $t \geq 0$,

$$
\begin{aligned}
\|z(t)\|^{2} & \leq e^{-t}\left\|u_{0}+v_{0}\right\|^{2}+C_{1}\left(v_{0}, t\right) \\
& +\frac{\left|d_{1}-d_{2}\right|^{2}}{d_{1} d_{2}} e^{-t}\left[\left(1+\frac{1}{2 \gamma d_{2}}\right) b^{2}|\Omega| e^{t}+\left(1+\alpha(t) e^{t}\right)\left\|v_{0}\right\|^{2}\right] \\
& \leq e^{-t}\left\|u_{0}+v_{0}\right\|^{2}+\alpha(t)\left\|v_{0}\right\|^{2}+\left(\frac{b^{2}}{\gamma d_{2}}+2 a^{2}\right)|\Omega| \\
& +\frac{\left|d_{1}-d_{2}\right|^{2}}{d_{1} d_{2}} e^{-t}\left[\left(1+\frac{1}{2 \gamma d_{2}}\right) b^{2}|\Omega| e^{t}+\left(1+\alpha(t) e^{t}\right)\left\|v_{0}\right\|^{2}\right]
\end{aligned}
$$

Note that (2.12) shows

$$
0<\alpha(t) \leq \max \left\{\frac{2}{\left|1-2 \gamma d_{2}\right|}, 4 e^{-1}\right\} e^{-\tilde{\alpha} t} \longrightarrow 0, \quad \text { as } t \rightarrow \infty,
$$

where

$$
\tilde{\alpha}=\min \left\{2 \gamma d_{2}, \frac{1}{2}\right\}>0 .
$$

Therefore, we can conclude from (2.14) that

$$
\limsup _{t \rightarrow \infty}\|z(t)\|^{2} \leq\left(\frac{b^{2}}{\gamma d_{2}}+2 a^{2}\right)|\Omega|+\frac{\left|d_{1}-d_{2}\right|^{2}}{d_{1} d_{2}}\left(1+\frac{1}{2 \gamma d_{2}}\right) b^{2}|\Omega|,
$$

The right-hand side of inequality (2.15) is a uniform positive constant, which is independent of any initial data. Let

$$
\rho_{1}=1+\left(\frac{b^{2}}{\gamma d_{2}}+2 a^{2}\right)|\Omega|+\frac{\left|d_{1}-d_{2}\right|^{2}}{d_{1} d_{2}}\left(1+\frac{1}{2 \gamma d_{2}}\right) b^{2}|\Omega| .
$$

Then we get

$$
\limsup _{t \rightarrow \infty}\|z(t)\|^{2}<\rho_{1}
$$


Putting together (2.3) and (2.16), we end up with

$$
\limsup _{t \rightarrow \infty}\|w(t)\|^{2}=\limsup _{t \rightarrow \infty}\left(\|z(t)-v(t)\|^{2}+\|v(t)\|^{2}\right)<K_{0}=2 \rho_{1}+3 \rho_{0} .
$$

Note that $K_{0}$ in (2.17) is a uniform positive constant independent of the initial data. Therefore the conclusion of this lemma is valid and we have shown the absorbing property of the Brusselator semiflow $\{S(t)\}_{t \geq 0}$ in the phase space $H$.

\section{Asymptotical Compactness by Decomposition}

For investigation of the asymptotical compactness for the Brusselator semiflow, we shall take the approach of showing the $\kappa$-contracting property for this semiflow $\{S(t)\}_{t \geq 0}$. Recall the definition of the Kuratowski measure of noncompactness for bounded sets in a Banach space $X$,

$\kappa(B) \stackrel{\text { def }}{=} \inf \{\delta: B$ has a finite cover by open sets in $X$ of diameters $<\delta\}$.

If $B$ is an unbounded set, then we define $\kappa(B)=\infty$. The basic properties of the Kuratowski measure are listed in the following lemma, cf. [24, Lemma 22.2] and [17, Lemma 2.4].

Lemma 4. Let $X$ be a Banach space and $\kappa$ be the Kuratowski measure of noncompactness of bounded sets in $X$. Then $\kappa$ has the following properties:

(i) $\kappa(B)=0$ if and only if $B$ is precompact in $X$, i.e. $C l_{X} B$ is a compact set in $X$.

(ii) $\kappa\left(B_{1}+B_{2}\right) \leq \kappa\left(B_{1}\right)+\kappa\left(B_{2}\right)$, for any linear sum $B_{1}+B_{2}$.

(iii) $\kappa\left(B_{1}\right) \leq \kappa\left(B_{2}\right)$ whenever $B_{1} \subset B_{2}$.

(iv) Suppose $X$ is a direct sum of two closed linear subspaces $X_{1}$ and $X_{2}$,

$$
X=X_{1} \oplus X_{2}, \quad \text { with } \operatorname{dim} X_{1}<\infty,
$$

and $P: X \rightarrow X_{1}$ and $Q: X \rightarrow X_{2}$ are the canonical projection operators. Let $B$ be a bounded set of $X$. If

$$
\operatorname{diam} Q(B)<\varepsilon
$$

then $\kappa(B)<\varepsilon$.

Definition 5. A semiflow $\{S(t)\}_{t \geq 0}$ on a complete metric space $X$ is called $\kappa$-contracting if for every bounded subset $B$ in $X$, one has

$$
\lim _{t \rightarrow \infty} \kappa(S(t) B)=0 \text {. }
$$

A semiflow $\{S(t)\}_{t \geq 0}$ on a complete metric space $X$ is called $\omega$-limit compact, if for every bounded subset $B$ of $X$, one has

$$
\lim _{t \rightarrow \infty} \kappa\left(\bigcup_{\tau \geq t} S(\tau) B\right)=0 .
$$

The following lemma provides the connection of the $\kappa$-contracting concept to the asymptotical compactness, cf. [24, Lemma 23.8] and Lemma 1. 
Lemma 5. Let $\{S(t)\}_{t \geq 0}$ be a semiflow on a Banach space $X$. If the following conditions are satisfied:

(i) $\{S(t)\}_{t \geq 0}$ has a bounded absorbing set in $X$, and

(ii) $\{S(t)\}_{t \geq 0}$ is $\kappa$-contracting,

then $\{S(t)\}_{t \geq 0}$ is asymptotically compact and there exists a global attractor $\mathcal{A}$ in $X$ for this semiflow.

Let us now try to make some a priori estimates to see what are the essential difficulties for an attempt to prove the $\kappa$-contracting property of the Brusselator semiflow. Taking the inner-product $\langle(1.2),-\Delta v(t)\rangle$, we have

$$
-\left\langle v_{t}, \Delta v\right\rangle+d_{2}\|\Delta v\|^{2}=\left\langle u^{2} v, \Delta v\right\rangle-b\langle u, \Delta v\rangle,
$$

By Green's formula and the homogeneous Dirichlet boundary condition, we obtain

$$
\begin{aligned}
& \frac{1}{2} \frac{d}{d t}\|\nabla v\|^{2}+d_{2}\|\Delta v\|^{2}=\int_{\Omega} u^{2} v \Delta v d x-b \int_{\Omega} u \Delta v d x \\
& \quad \leq \int_{\Omega} u^{2} v \Delta v d x+c\|u\|^{2}+\frac{d_{2}}{2}\|\Delta v\|^{2} \\
& \quad=-\int_{\Omega} u^{2}|\nabla v|^{2} d x-2 \int_{\Omega} u v(\nabla u \cdot \nabla v) d x+c\|u\|^{2}+\frac{d_{2}}{2}\|\Delta v\|^{2} \\
& \quad=-\int_{\Omega}|u \nabla v+v \nabla u|^{2} d x+\int_{\Omega} v^{2}|\nabla u|^{2} d x+c\|u\|^{2}+\frac{d_{2}}{2}\|\Delta v\|^{2} \\
& \quad \leq \int_{\Omega} v^{2}|\nabla u|^{2} d x+c\|u\|^{2}+\frac{d_{2}}{2}\|\Delta v\|^{2},
\end{aligned}
$$

where $c$ is a positive constant depending only on $b$ and $d_{2}$. Consequently we get

$$
\begin{aligned}
\frac{d}{d t}\|\nabla v\|^{2}+d_{2}\|\Delta v\|^{2} & \leq 2 \int_{\Omega} u^{2} v \Delta v d x+2 c\|u\|^{2} \\
& \leq 2 \int_{\Omega} v^{2}|\nabla u|^{2} d x+2 c\|u\|^{2} .
\end{aligned}
$$

The troubling terms are the integrals on the right-hand side of (3.1) and (3.2), and much difficulty and complexity occur in this kind of estimation for $u(t)$ or $z(t)$. As commented in Section 1, these difficulties originate from the lack of dissipativity in the structural polynomial nonlinearity of the Brusselator equations.

A generic and good idea in dealing with the issue of proving the asymptotical compactness and the $\kappa$-contracting property is through a decomposition approach. We have seen different decomposition methods in different settings.

In many papers addressing the existence of global attractors for nonlinear wave equations and other nonlinear hyperbolic evolutionary equations, the solution semigroup $S(t)$ is decomposed into two parts, $S(t)=S_{1}(t)+S_{2}(t)$, where $S_{1}(t) B$ uniformly converges to zero as $t \rightarrow \infty$ and $S_{2}(t) B$ is precompact or $\kappa$-contracting for any bounded set $B$, cf. $[\mathbf{2 4}, \mathbf{2 7}, \mathbf{3 5}]$ and references therein.

Another decomposition method featuring smallness of tail estimates was introduced in $[4,30]$ and effectively used in search for global attractors both for the first-order and for the second-order lattice and PDE systems. Combined with the Littlewood-Paley projection techniques of harmonic analysis, such a decomposition 
with tail estimation was applied to the asymptotic smoothing and the existence of global attractors for the generalized Benjamin-Bona-mahony equations on $\Re^{3}$ in $[\mathbf{2 5}]$.

The spectral decomposition [17] and the spatial cut-off decomposition [28] were also introduced to address the existence of global attractors for certain classes of scalar nonlinear reaction-diffusion equations in $L^{p}$ spaces, or on unbounded domains.

Here we present a new decomposition method, which is stated in the next theorem. In the next section, we shall check the $\kappa$-contracting property and the asymptotical compactness of the Brusselator semiflow by this method.

We shall use the notation $\Omega(|u| \geq M)=\{x \in \Omega:|u(x)| \geq M\}$ and $\Omega(|u|<$ $M)=\{x \in \Omega:|u(x)|<M\}$, and use $m(\cdot)$ to denote the Lebesgue measure of a subset in $\Omega$.

Theorem 2. Let $Y=L^{2}(\Omega)$ or $H$. Let $\{S(t)\}_{t \geq 0}$ be a semiflow on $Y$. Then there exists a global attractor $\mathcal{A}$ in $Y$ for this semiflow if and only if the following two conditions are satisfied :

(i) There exists a bounded absorbing set $B_{0}$ in $Y$ for this semiflow.

(ii) For any $\varepsilon>0$, there are positive constants $M=M(\varepsilon)$ and $T=T(\varepsilon)$ such that

$$
\int_{\Omega\left(\left|S(t) w_{0}\right| \geq M\right)}\left|S(t) w_{0}\right|^{2} d x<\varepsilon, \quad \text { for any } t \geq T, w_{0} \in B_{0} .
$$

and

$$
\kappa\left(\left(S(t) B_{0}\right)_{\Omega\left(\left|S(t) B_{0}\right|<M\right)}\right) \longrightarrow 0, \text { as } t \rightarrow \infty
$$

where

$$
\left(S(t) B_{0}\right)_{\Omega\left(\left|S(t) B_{0}\right|<M\right)} \stackrel{\text { def }}{=}\left\{\left(S(t) w_{0}\right)(\cdot) \theta_{M}\left(\cdot ; t, w_{0}\right): \text { for } w_{0} \in B_{0}\right\} .
$$

and $\theta_{M}\left(x ; t, w_{0}\right)$ is the characteristic function of the subset $\Omega\left(\left|S(t) w_{0}\right|<M\right)$.

Proof. First we prove the sufficiency. By the condition (i), according to Lemma 5, it suffices to show that $\{S(t)\}_{t \geq 0}$ is $\kappa$-contracting, i.e.

$$
\lim _{t \rightarrow \infty} \kappa(S(t) B)=0, \quad \text { for any bounded set } B \subset Y \text {. }
$$

Since $B_{0}$ attracts any bounded set $B$, there is a time $T_{B}$ such that $S(t) B \subset B_{0}$, for $t \geq T_{B}$. Therefore, it suffices to show that

$$
\lim _{t \rightarrow \infty} \kappa\left(S(t) B_{0}\right)=0,
$$

for the absorbing set $B_{0}$. By a property of the Kuratowski measure,

$$
\kappa\left(B_{1}+B_{2}\right) \leq \kappa\left(B_{1}\right)+\kappa\left(B_{2}\right)
$$

we see that

$$
\kappa\left(S(t) B_{0}\right) \leq \kappa\left(S(t) B_{0}\left(1-\theta_{M}\right)\right)+\kappa\left(S(t) B_{0} \theta_{M}\right),
$$

where $S(t) B_{0} \theta_{M}$ represents the set

$$
S(t) B_{0} \theta_{M} \stackrel{\text { def }}{=}\left\{\left(S(t) w_{0}\right) \cdot \theta_{M}\left(\cdot ; t, w_{0}\right) \text { : for all } w_{0} \in B_{0}\right\},
$$

and similarly for $S(t) B_{0}\left(1-\theta_{M}\right)$. Now (3.3) in the condition (ii) indicates that for any $\varepsilon>0$, there exist positive constants $M=M(\varepsilon)$ and $T=(\varepsilon)$ such that 


$$
\int_{\Omega}\left|S(t) w_{0}\left(1-\theta_{M}\left(x ; t, w_{0}\right)\right)\right|^{2} d x=\int_{\Omega\left(\left|S(t) w_{0}\right| \geq M\right)}\left|S(t) w_{0}\right|^{2} d x<\frac{\varepsilon^{2}}{4},
$$

for $t \geq T(\varepsilon), w_{0} \in B_{0}$. Then (3.7) implies that

$$
\kappa\left(S(t) B_{0}\left(1-\theta_{M}\right)\right)<\varepsilon, \quad \text { for } t \geq T(\varepsilon) .
$$

On the other hand, by (3.4), for the same arbitrary $\varepsilon$ and the same $M=M(\varepsilon)$, there exists a time $T_{0}\left(\varepsilon, B_{0}\right)>0$ such that

$$
\kappa\left(S(t) B_{0} \theta_{M}\right)=\kappa\left(S(t)\left(B_{0}\right)_{\Omega\left(\left|S(t) B_{0}\right|<M\right)}\right)<\varepsilon, \quad \text { for } t \geq T_{0}\left(\varepsilon, B_{0}\right) .
$$

Substituting the above two inequalities into (3.6), we obtain

$$
\kappa\left(S(t) B_{0}\right)<2 \varepsilon, \quad \text { for any } t \geq T(\varepsilon)+T_{0}\left(\varepsilon, B_{0}\right) .
$$

Therefore, (3.5) is proved and $\{S(t)\}_{t \geq 0}$ is $\kappa$-contracting. By Lemma 5 there exists a global attractor for this semiflow.

Now we prove the necessity. We check each of the two conditions stated in this theorem. Suppose there exists a global attractor $\mathcal{A}$ in $Y$ for this semiflow. Then $\mathcal{A}$ is a compact set. Given a constant $\varepsilon_{0}>0$, then for every bounded set $B \subset Y$ there is a time $t_{B}$ such that

$$
S(t) B \subset N_{\varepsilon_{0}}(\mathcal{A}), \quad \text { for } t \geq t_{B},
$$

where the neighborhood $N_{\varepsilon_{0}}(\mathcal{A})$ of $\mathcal{A}$ is a bounded absorbing set. Thus the condition (i) is satisfied.

To show the condition (ii) is satisfied, we have the following observations. First, since $\operatorname{dist}\left(S(t) B_{0}, \mathcal{A}\right) \rightarrow 0$, as $t \rightarrow \infty$, for any given $\varepsilon>0$, there is a $T^{*}>0$ such that

$$
\operatorname{dist}\left(S(t) B_{0}, \mathcal{A}\right) \leq\left(\frac{\varepsilon}{6}\right)^{1 / 2}, \quad \text { for } t \geq T^{*} .
$$

Since it is a compact set in $Y$, there is a finite covering net $\left\{\varphi_{1}, \cdots, \varphi_{r}\right\} \subset \mathcal{A}$ with the property that for any $\varphi \in \mathcal{A}$, there is a $\varphi_{i} \in\left\{\varphi_{1}, \cdots, \varphi_{r}\right\}$ such that

$$
\left\|\varphi-\varphi_{i}\right\|^{2}<\frac{\varepsilon}{6}
$$

By the absolute continuity of the Lebesgue integral, for this arbitrarily given $\varepsilon>0$, there is an $\eta=\eta(\varepsilon)>0$ such that for any measurable set $\Omega_{s} \subset \Omega$ satisfying $m\left(\Omega_{s}\right)<\eta$, one has

$$
\int_{\Omega_{s}}\left|\varphi_{i}(x)\right|^{2} d x<\frac{\varepsilon}{6}, \quad \text { for } i=1, \cdots, r .
$$

Note that for every $w_{0} \in B_{0}$, there exists a uniform positive constant $T\left(B_{0}\right)$ such that $\left\|S(t) w_{0}\right\|^{2} \leq K_{0}$, for $t \geq T\left(B_{0}\right)$, where $K_{0}$ is the uniform constant given by (2.17). Thus there is a large $M=M(\varepsilon)>0$ such that

$$
m\left(\Omega\left(\left|S(t) w_{0}\right| \geq M\right) \leq \frac{\left\|S(t) w_{0}\right\|^{2}}{M^{2}} \leq \frac{K_{0}}{M^{2}}<\eta, \quad \text { for any } w_{0} \in B_{0} .\right.
$$


Putting all together, we obtain that for this $M=M(\varepsilon)$,

$$
\begin{aligned}
\int_{\Omega\left(\left|S(t) w_{0}\right| \geq M\right)}\left|S(t) w_{0}\right|^{2} d x & \leq 2 \int_{\Omega\left(\left|S(t) w_{0}\right| \geq M\right)}\left|S(t) w_{0}-\varphi\right|^{2} d x \\
& +2 \int_{\Omega\left(\left|S(t) w_{0}\right| \geq M\right)}\left|\varphi(x)-\varphi_{i_{0}}\right|^{2} d x \\
& +2 \int_{\Omega\left(\left|S(t) w_{0}\right| \geq M\right)}\left|\varphi_{i_{0}}\right|^{2} d x \\
& <\frac{\varepsilon}{3}+\frac{\varepsilon}{3}+\frac{\varepsilon}{3}=\varepsilon, \quad \text { for } t \geq T,
\end{aligned}
$$

where $\varphi$ is some point in $\mathcal{A}, i_{0} \in\{1, \cdots, r\}$, and $T=\max \left\{T^{*}, T\left(B_{0}\right)\right\}$. Therefore, it is proved that (3.3) in the condition (ii) is satisfied by this semiflow.

Now we verify (3.4) in the condition (ii). By [17, Theorem 3.9], the existence of a global attractor $\mathcal{A}$ in $Y$ implies that the semiflow $\{S(t)\}_{t \geq 0}$ is $\omega$-limit compact, which by Definition 5 implies that the semiflow $\{S(t)\}_{t \geq 0}$ is $\kappa$-contracting. Hence, for the bounded absorbing set $B_{0}$ in $Y$, we have

$$
\kappa\left(S(t) B_{0}\right) \rightarrow 0, \quad \text { as } t \rightarrow \infty .
$$

By the definition of the $\theta_{M}$ functions, we have

$$
\kappa\left(S(t) B_{0} \theta_{M}\right) \leq \kappa\left(S(t) B_{0}\right)+\kappa\left(S(t) B_{0}\left(1-\theta_{M}\right)\right) .
$$

For any $\varepsilon>0$, there exists $\widetilde{T}(\varepsilon)>0$ such that $\kappa\left(S(t) B_{0}\right)<\varepsilon$. whenever $t \geq \widetilde{T}(\varepsilon)$. On the other hand, we have shown in the verification of (3.3) that for every $w_{0} \in B_{0}$,

$$
\int_{\Omega}\left|\left(S(t) w_{0}\right)(x)\left(1-\theta_{M}\left(x ; t, w_{0}\right)\right)\right|^{2} d x<\varepsilon, \quad \text { for } t \geq T,
$$

where $T$ is shown in (3.8). This implies that

$$
\kappa\left(S(t) B_{0}\left(1-\theta_{M}\right)\right)<2 \varepsilon,
$$

and, consequently,

$$
\kappa\left(S(t) B_{0} \theta_{M}\right)<3 \varepsilon, \quad \text { for } t \geq \widehat{T}=\max \{\widetilde{T}(\varepsilon), T\} .
$$

This shows that (3.4) in the condition (ii) is valid.

Similarly we can prove the following theorem which gives another set of necessary and sufficient conditions for the existence of a global attractor in this case and will be used to show the $\kappa$-contracting property of the Brusselator semiflow $\{S(t)\}_{t \geq 0}$.

TheOREM 3. For the Brusselator semiflow $\{S(t)\}_{t \geq 0}$ on $H$, there exists a global attractor $\mathcal{A}$ in $H$ if and only if the following two conditions are satisfied:

(i) There exists a bounded absorbing set $B_{0}$ in $H$ for this semiflow.

(ii) For any $\varepsilon>0$, there are positive constants $M=M(\varepsilon)$ and $T=T(\varepsilon)$ such that

$$
\int_{\Omega(|v(t)| \geq M)}\left|S(t) w_{0}\right|^{2} d x<\varepsilon, \quad \text { for any } t \geq T, w_{0} \in B_{0}
$$


and

$$
\kappa\left(\left(S(t) B_{0}\right)_{\Omega(|v(t)|<M)}\right) \longrightarrow 0, \text { as } t \rightarrow \infty
$$

where

$$
\left(S(t) B_{0}\right)_{\Omega(|v(t)|<M)} \stackrel{\text { def }}{=}\left\{\left(S(t) w_{0}\right)(\cdot) \zeta_{M}\left(\cdot ; t, w_{0}\right): \text { for } w_{0} \in B_{0}\right\},
$$

in which $\zeta_{M}\left(x ; t, w_{0}\right)$ is the characteristic function of the subset $\Omega(|v(t)|<M)$, and $v(t)=v\left(t, x, w_{0}\right)$ is the $v$-component of the solution of the Brusselator equations $(1.1)-(1.2)$.

The proof of Theorem 3 is just parallel to the proof of Theorem 2 and is omitted. Note that in Theorem 2 and in Theorem 3 the condition (ii) corresponds to the $\kappa$-contracting property.

\section{4. $\kappa$-Contracting Property}

In this section, we shall check that the conditions in Theorem 3 are satisfied by the Brusselator semiflow. Through this decomposition approach, the existence of a global attractor for this Brusselator semiflow $\{S(t)\}_{t \geq 0}$ will be shown.

LEMmA 6. For any $\varepsilon>0$, there exist positive constants $M_{1}=M_{1}(\varepsilon)$ and $T_{1}=T_{1}(\varepsilon)$ such that the $v$-component of the solution of the Brusselator equations $(1.1)-(1.2), v(t)=v\left(t, x, w_{0}\right)$, satisfies

$$
\int_{\Omega\left(|v(t)| \geq M_{1}\right)}|v(t)|^{2} d x<\frac{2 b^{2}}{d_{2} \gamma} \varepsilon, \quad \text { for } t \geq T_{1}, w_{0}=\left(u_{0}, v_{0}\right) \in B_{0} .
$$

Proof. First of all, since $B_{0}$ is a bounded absorbing set, there exist uniform constants $T_{0}=T_{0}\left(B_{0}\right)>0$ and $K_{0}>0$, where $K_{0}$ is given in (2.17), such that

$$
\|v(t)\|^{2}=\left\|v\left(t, \cdot, w_{0}\right)\right\|^{2} \leq\left\|S(t) w_{0}\right\|^{2} \leq K_{0}, \quad \text { for } t \geq T_{0}, w_{0}=\left(u_{0}, v_{0}\right) \in B_{0}
$$

Hence we have

$$
M^{2} m(\Omega(|v(t)| \geq M)) \leq \int_{\Omega(|v(t)| \geq M)}\left|S(t) w_{0}\right|^{2} d x \leq K_{0},
$$

so that there exists an $M=M(\varepsilon)>0$ such that

$$
m(\Omega(|v(t)| \geq M)) \leq \frac{K_{0}}{M^{2}}<\frac{\varepsilon}{2}, \quad \text { for } t \geq T_{0}, w_{0} \in B_{0}
$$

Taking the inner-product $\left\langle(1.2),(v(t)-M)_{+}\right\rangle$, where $M$ is given in (4.2) and

$$
(\varphi-M)_{+}= \begin{cases}\varphi(x)-M, & \text { if } \varphi(x) \geq M, \\ 0, & \text { if } \varphi(x)<M,\end{cases}
$$


we obtain

$$
\begin{aligned}
& \frac{1}{2} \frac{d}{d t}\left\|(v-M)_{+}\right\|^{2}+d_{2} \int_{\Omega(v(t) \geq M)}\left|\nabla(v-M)_{+}\right|^{2} d x \\
& =-\int_{\Omega(v(t) \geq M)} u^{2} v(v-M)_{+} d x+\int_{\Omega(v(t) \geq M)} b u(v-M)_{+} d x \\
& \leq-\int_{\Omega(v(t) \geq M)}\left[\left(u(v-M)_{+}-\frac{b}{2}\right)^{2}+u^{2} M(v-M)_{+}\right] d x \\
& +\frac{b^{2}}{4} m(\Omega(v(t) \geq M)) \leq \frac{b^{2}}{4} \cdot \frac{\varepsilon}{2}=\frac{b^{2}}{8} \varepsilon .
\end{aligned}
$$

It follows that

$$
\frac{d}{d t}\left\|(v-M)_{+}\right\|^{2}+2 d_{2} \gamma\|v(t)-M\|^{2} \leq \frac{b^{2}}{4} \varepsilon
$$

and, by the Gronwall inequality,

$$
\left\|(v(t)-M)_{+}\right\|^{2} \leq e^{-2 d_{2} \gamma t}\left\|\left(v_{0}-M\right)_{+}\right\|^{2}+\frac{b^{2} \varepsilon}{8 d_{2} \gamma} .
$$

Thus there exists a time $T_{+}(\varepsilon, M)$ such that for any $t \geq T_{+}$and any $w_{0} \in B_{0}$, one has

$$
\left\|(v(t)-M)_{+}\right\|^{2}=\int_{\Omega(v(t) \geq M)}|v(t)-M|^{2} d x<\frac{b^{2} \varepsilon}{4 d_{2} \gamma} .
$$

Symmetrically we can prove that there exists a time $T_{-}(\varepsilon, M)$ such that for any $t \geq T_{-}$and any $w_{0} \in B_{0}$, one has

$$
\left\|(v(t)+M)_{-}\right\|^{2}=\int_{\Omega(v(t) \leq-M)}|v(t)+M|^{2} d x<\frac{b^{2} \varepsilon}{4 d_{2} \gamma},
$$

where

$$
(\varphi+M)_{-}= \begin{cases}\varphi(x)+M, & \text { if } \varphi(x) \leq-M \\ 0, & \text { if } \varphi(x)>-M\end{cases}
$$

Therefore, for any $t \geq T_{1}(\varepsilon)=\max \left\{T_{+}, T_{-}\right\}$, and for any $w_{0} \in B_{0}$, there holds

$$
\int_{\Omega(|v(t)| \geq M)}(|v(t)|-M)^{2} d x<\frac{b^{2} \varepsilon}{2 d_{2} \gamma} .
$$

Next we can deduce that there exists a positive integer $k$ such that

$$
\int_{\Omega(|v(t)| \geq k M)}|v(t)|^{2} d x<\frac{2 b^{2} \varepsilon}{d_{2} \gamma} .
$$

Indeed, referring to (4.2), we see that for any $w_{0} \in B_{0}$,

$$
m(\Omega(|v(t)| \geq k M)) \leq \frac{K_{0}}{k^{2} M^{2}}
$$


where $K_{0} / M^{2}<\varepsilon / 2$. Then it follows that

$$
\begin{aligned}
\int_{\Omega(|v(t)| \geq k M)}|v(t)|^{2} d x & \leq 2 \int_{\Omega(|v(t)| \geq M)}(|v(t)|-M)^{2} d x+2 M^{2} m(\Omega(|v(t)| \geq k M)) \\
& \leq \frac{b^{2} \varepsilon}{d_{2} \gamma}+\frac{2 M^{2} K_{0}}{k^{2} M^{2}}=\frac{b^{2} \varepsilon}{d_{2} \gamma}+\frac{2 K_{0}}{k^{2}}<\frac{2 b^{2} \varepsilon}{d_{2} \gamma}
\end{aligned}
$$

for a sufficiently large integer $k(\varepsilon)$. Therefore (4.7) holds and, consequently, (4.1) is proved with $M_{1}=M_{1}(\varepsilon)=k M$, where $M$ is given in (4.2), and $T_{1}=T_{1}(\varepsilon)=$ $\max \left\{T_{+}, T_{-}\right\}$, for any $w_{0}=\left(u_{0}, v_{0}\right) \in B_{0}$.

Lemma 7. Let $P_{v}: H \rightarrow L^{2}(\Omega)_{v}$ be the orthogonal projection from the product Hilbert space $H$ onto the second component space associated with the $v$-component. Let $B_{0}$ be the bounded absorbing set of the Brusselator semiflow $\{S(t)\}_{t \geq 0}$ in $H$, shown in Lemma 3. Then for any given $M>0$, it holds that

$$
\kappa\left(P_{v}\left(S(t) B_{0}\right)_{\Omega(|v(t)|<M)}\right) \longrightarrow 0, \text { as } t \rightarrow \infty
$$

where $\left(S(t) B_{0}\right)_{\Omega(|v(t)|<M)}$ has been specified in Theorem 3 .

Proof. Let us consider the inequality similar to (3.2) but with integrals over the set $\Omega_{v, M}=\Omega(|v(t)|<M)$, namely,

$$
\frac{d}{d t}\|\nabla v\|_{\Omega_{v, M}}^{2}+d_{2}\|\Delta v\|_{\Omega_{v, M}}^{2} \leq 2 \int_{\Omega_{v, M}} v^{2}|\nabla u|^{2} d x+2 c\|u\|_{\Omega_{v, M}}^{2},
$$

where the $L^{2}\left(\Omega_{v, M}\right)$-norm is denoted by $\|\cdot\|_{\Omega_{v, M}}$. Consequently, we have

$$
\frac{d}{d t}\|\nabla v\|_{\Omega_{v, M}}^{2}+d_{2} \gamma\|\nabla v\|_{\Omega_{v, M}}^{2} \leq 2 M^{2}\|\nabla u\|_{\Omega_{v, M}}^{2}+2 c K_{0},
$$

for any $w_{0} \in B_{0}$ and any $t \geq T_{0}=T_{0}\left(B_{0}\right)$, where $K_{0}$ is given by $(2.17)$ and $T_{0}$ is given in the beginning of the proof of Lemma 6. Inequality (4.10) implies that

$$
\frac{d y}{d t} \leq g y+h, \quad t>0
$$

where

$$
\begin{aligned}
& y(t)=\|\nabla v(t)\|_{\Omega_{v, M}}^{2}, \quad g(t)=d_{2} \gamma, \quad \text { and } \\
& h(t)=2 M^{2}\|\nabla u\|_{\Omega_{v, M}}^{2}+2 c K_{0} .
\end{aligned}
$$

From (2.4) now over $\Omega_{v, M}$ we see that there exists a constant $T_{2}=T_{2}\left(B_{0}\right)>0$, such that

$$
\int_{t}^{t+1}\|\nabla v(s)\|_{\Omega_{v, M}}^{2} d s \leq C_{2}(M)=\frac{b^{2}\left|\Omega_{v, M}\right|}{d_{2}}\left(\frac{1}{\gamma d_{2}}+1\right),
$$

for $t \geq T_{2}, w_{0} \in B_{0}$. By integrating the inequality corresponding to (2.6) now over $\Omega_{v, M}$ on the time interval $[t, t+1]$, and using (4.12), we can deduce that there exists a constant $T_{3}=T_{3}\left(B_{0}\right)$, with $T_{3} \geq \max \left\{T_{0}\left(B_{0}\right), T_{2}\left(B_{0}\right)\right\}$, such that 


$$
d_{1} \int_{t}^{t+1}\|\nabla z(s)\|_{\Omega_{v, M}}^{2} d s \leq \frac{\left|d_{1}-d_{2}\right|^{2}}{d_{1}} C_{2}(M)+2\left(\frac{b^{2}}{\gamma d_{2}}+2 a^{2}\right)\left|\Omega_{v, M}\right|+2 K_{0},
$$

for $t \geq T_{3}$, which implies that

$$
\begin{aligned}
\int_{t}^{t+1}\|\nabla u(s)\|_{\Omega_{v, M}}^{2} d s & =\int_{t}^{t+1}\|\nabla z(s)-\nabla v(s)\|_{\Omega_{v, M}}^{2} d s \\
& \leq 2 \int_{t}^{t+1}\left(\|\nabla z(s)\|_{\Omega_{v, M}}^{2}+\|\nabla v(s)\|_{\Omega_{v, M}}^{2}\right) d s \\
& \leq C_{3}(M), \quad \text { for } t \geq T_{3}, \quad w_{0} \in B_{0},
\end{aligned}
$$

where

$$
C_{3}(M)=2\left[C_{2}(M)\left(1+\frac{\left|d_{1}-d_{2}\right|^{2}}{d_{1}^{2}}\right)+\frac{2}{d_{1}}\left(\frac{b^{2}}{\gamma d_{2}}+2 a^{2}\right)\left|\Omega_{v, M}\right|+\frac{2 K_{0}}{d_{1}}\right] .
$$

This yields that

$$
\int_{t}^{t+1} h(s) d s \leq 2 M^{2} C_{3}(M)+2 c K_{0}, \quad \text { for } t \geq T_{3}, w_{0} \in B_{0}
$$

Obviously,

$$
\int_{t}^{t+1} g(s) d s \leq d_{2} \gamma
$$

Finally by inequalities (4.12), (4.14) and (4.15), and applying the uniform Gronwall inequality $[\mathbf{2 4}, \mathbf{2 9}]$ to $(4.11)$, we can conclude that the following inequality holds,

$$
\|\nabla v(t)\|_{\Omega_{v, M}}^{2} \leq\left(2 M^{2} C_{3}(M)+2 c K_{0}+C_{2}(M)\right) e^{d_{2} \gamma} \quad \text { for any } t \geq T_{3}+1, w_{0} \in B_{0} .
$$

Note that the right-hand side of (4.16) is a uniform constant depending on the absorbing set $B_{0}$ and the arbitrarily given $M$ only.

The inequality (4.16) shows that for any fixed $t \geq T_{3}+1$,

$$
P_{v}\left(S(t) B_{0}\right)_{\Omega(|v(t)|<M)} \text { is a bounded set in } H_{0}^{1}(\Omega) \text {. }
$$

Due to the compact Sobolev embedding $H_{0}^{1}(\Omega) \hookrightarrow L^{2}(\Omega)$ for $n \leq 3$, it turns out that for any fixed $t \geq T_{3}+1$,

$$
P_{v}\left(S(t) B_{0}\right)_{\Omega(|v(t)|<M)} \text { is a precompact set in } L^{2}(\Omega) \text {. }
$$

Therefore, by Lemma 4 it is proved that, in the space $L^{2}(\Omega)$,

$$
\kappa\left(P_{v}\left(S(t) B_{0}\right)_{\Omega(|v(t)|<M)}\right)=0, \quad \text { for } t \geq T_{3}\left(B_{0}\right)+1 \text {. }
$$

This shows that (4.9) is valid for any given $M>0$. The lemma is proved.

Next we treat the $u$-component through $z(t)=u(t)+v(t)$. We shall use the notation

$$
\Omega_{M}^{v}=\Omega(|v(t)| \geq M) .
$$


Lemma 8. For any $\varepsilon>0$, there exist positive constants $M_{2}=M_{2}(\varepsilon)$ and $\tau_{2}=$ $\tau_{2}(\varepsilon)$ such that the u-component of the solution of the Brusselator equations (1.1)(1.2) with the initial datum $w_{0}$ satisfies

$$
\int_{\Omega_{M_{2}}^{v}}|u(t)|^{2} d x<\Gamma_{2} \varepsilon, \quad \text { for } t \geq \tau_{2}, w_{0} \in B_{0},
$$

where $\Gamma_{2}>0$ is a uniform constant independent of the initial datum $w_{0} \in B_{0}$.

Proof. For any $w_{0} \in B_{0}$, according to (4.2), we have

$$
m(\Omega(|v(t)| \geq M)) \leq \frac{K_{0}}{M^{2}}<\frac{\varepsilon}{2}, \quad \text { for } t>T_{0} .
$$

Let $z(t)=u\left(t, x, w_{0}\right)+v\left(t, x, w_{0}\right)$, where $(u, v)$ is the solution of the Brusselator equations with the initial datum $w_{0}$. Taking the inner-product $\langle(2.5), z(t)\rangle_{\Omega_{M}^{v}}$ over the set $\Omega_{M}^{v}$, we get

$$
\begin{aligned}
& \frac{1}{2} \frac{d}{d t}\|z(t)\|_{\Omega_{M}^{v}}^{2}+d_{1}\|\nabla z(t)\|_{\Omega_{M}^{v}}^{2}+\|z(t)\|_{\Omega_{M}^{v}}^{2} \\
& \quad=\int_{\Omega_{M}^{v}}\left(d_{2}-d_{1}\right) z(t) \Delta v(t) d x+\int_{\Omega_{M}^{v}}(v(t)+a) z(t) d x, \\
& \quad \leq \frac{d_{1}}{2}\|\nabla z(t)\|_{\Omega_{M}^{v}}^{2}+\frac{\left|d_{1}-d_{2}\right|^{2}}{2 d_{1}}\|\nabla v(t)\|_{\Omega_{M}^{v}}^{2}+\frac{1}{2}\|z(t)\|_{\Omega_{M}^{v}}^{2}+\frac{1}{2}\|v(t)+a\|_{\Omega_{M}^{v}}^{2},
\end{aligned}
$$

from which we can get

$$
\begin{aligned}
\frac{d}{d t}\|z(t)\|_{\Omega_{M}^{v}}^{2} & +d_{1}\|\nabla z(t)\|_{\Omega_{M}^{v}}^{2}+\|z(t)\|_{\Omega_{M}^{v}}^{2} \\
& \leq \frac{\left|d_{1}-d_{2}\right|^{2}}{d_{1}}\|\nabla v(t)\|_{\Omega_{M}^{v}}^{2}+\|v(t)+a\|_{\Omega_{M}^{v}}^{2} \\
& \leq \frac{\left|d_{1}-d_{2}\right|^{2}}{d_{1}}\|\nabla v(t)\|_{\Omega_{M}^{v}}^{2}+2\|v(t)\|_{\Omega_{M}^{v}}^{2}+2 a^{2}\left|\Omega_{M}^{v}\right| .
\end{aligned}
$$

It follows that

$$
\frac{d}{d t}\left(e^{t}\|z(t)\|_{\Omega_{M}^{v}}^{2}\right) \leq \frac{\left|d_{1}-d_{2}\right|^{2}}{d_{1}} e^{t}\|\nabla v(t)\|_{\Omega_{M}^{v}}^{2}+e^{t}\left(2\|v(t)\|_{\Omega_{M}^{v}}^{2}+2 a^{2}\left|\Omega_{M}^{v}\right|\right) .
$$

Integrating both sides of this inequality on $[0, t]$, we obtain

$$
\begin{aligned}
\|z(t)\|_{\Omega_{M}^{v}}^{2} & =\int_{\Omega_{M}^{v}}|z(t)|^{2} d x \leq e^{-t}\left\|u_{0}+v_{0}\right\|_{\Omega_{M}^{v}}^{2} \\
& +\frac{\left|d_{1}-d_{2}\right|^{2}}{d_{1}} e^{-t} \int_{0}^{t} e^{s}\|\nabla v(s)\|_{\Omega_{M}^{v}}^{2} d s \\
& +e^{-t} \int_{0}^{t} e^{s}\left(2\|v(s)\|_{\Omega_{M}^{v}}^{2}+2 a^{2}\left|\Omega_{M}^{v}\right|\right) d s .
\end{aligned}
$$

Similar to (2.13), we have

$$
d_{2} \int_{0}^{t} e^{s}\|\nabla v(s)\|_{\Omega_{M}^{v}}^{2} d s \leq\left(1+\frac{1}{2 d_{2} \gamma}\right) b^{2}\left|\Omega_{M}^{v}\right| e^{t}+\left(1+\alpha(t) e^{t}\right) K_{0},
$$


where $K_{0}$ is the uniform constant given in (2.17). Then we can estimate the terms on the right-hand side of the inequality (4.19) as follows. For sufficiently large $t>0$, there holds

$$
e^{-t}\left\|u_{0}+v_{0}\right\|_{\Omega_{M}^{v}}^{2} \leq 2 K_{0} e^{-t}<\varepsilon .
$$

Since $\left|\Omega_{M}^{v}\right|<\varepsilon / 2$ and $\alpha(t) \rightarrow 0$ as $t \rightarrow \infty$, (4.20) implies that, for sufficiently large $t>0$,

$$
\begin{aligned}
e^{-t} & \frac{\left|d_{1}-d_{2}\right|^{2}}{d_{1}} \int_{0}^{t} e^{s}\|\nabla v(s)\|_{\Omega_{M}^{v}}^{2} d s \\
& \leq \frac{\left|d_{1}-d_{2}\right|^{2}}{d_{1} d_{2}}\left[\left(1+\frac{1}{2 d_{2} \gamma}\right) b^{2}\left|\Omega_{M}^{v}\right|+\left(e^{-t}+\alpha(t)\right) K_{0}\right] \\
& <\frac{\left|d_{1}-d_{2}\right|^{2}}{d_{1} d_{2}}\left(1+\frac{1}{2 d_{2} \gamma}\right) b^{2} \varepsilon .
\end{aligned}
$$

Moreover, for sufficientlt large $t>0$,

$$
e^{-t} \int_{0}^{t} e^{s}\|v(s)\|_{\Omega_{M}^{v}}^{2} d s \leq e^{-t} \int_{0}^{t} \frac{e^{s}}{\gamma}\|\nabla v(s)\|_{\Omega_{M}^{v}}^{2} d s<\frac{1}{d_{2} \gamma}\left(1+\frac{1}{2 d_{2} \gamma}\right) b^{2} \varepsilon,
$$

and

$$
e^{-t} \int_{0}^{t} e^{s} 2 a^{2}\left|\Omega_{M}^{v}\right| d s<2 a^{2}\left|\Omega_{M}^{v}\right|<a^{2} \varepsilon
$$

Finally, substitute these estimates into (4.19), we find that there exists a time $\tau_{1}=\tau_{1}(\varepsilon, M)$ such that

$$
\begin{aligned}
\|z(t)\|_{\Omega_{M}^{v}}^{2} & =\int_{\Omega(|v(t)| \geq M)}|z(t)|^{2} d x \\
& \leq \varepsilon\left(1+a^{2}\right)+\left(\frac{\left|d_{1}-d_{2}\right|^{2}}{d_{1} d_{2}}+\frac{1}{d_{2} \gamma}\right)\left(1+\frac{1}{2 d_{2} \gamma}\right) b^{2} \varepsilon \\
& =\Gamma_{1} \varepsilon, \quad \text { for } t \geq \tau_{1}, w_{0} \in B_{0},
\end{aligned}
$$

where $\Gamma_{1}$ is a uniform constant. Combining (4.21) with (4.1),

$$
\|v(t)\|_{\Omega_{M_{1}}^{v}}^{2}<\frac{2 b^{2}}{d_{2} \gamma} \varepsilon, \quad \text { for } t \geq T_{1},
$$

we see that there exist positive constants

$$
M_{2}=\max \left\{M, M_{1}\right\}, \quad \Gamma_{2}=2\left(\Gamma_{1}+\frac{2 b^{2}}{d_{2} \gamma}\right), \quad \tau_{2}=\max \left\{T_{1}, \tau_{1}\right\},
$$

such that

$$
\int_{\Omega\left(|v(t)| \geq M_{2}\right)}|u(t)|^{2} d x \leq 2\left(\|v(t)\|_{\Omega_{M_{1}}^{v}}^{2}+\|z(t)\|_{\Omega_{M}^{v}}^{2}\right)<\Gamma_{2} \varepsilon,
$$

for $t \geq \tau_{2}, w_{0}=\left(u_{0}, v_{0}\right) \in B_{0}$. Therefore, (4.17) is proved.

Lemma 9. Let $P_{u}: H \rightarrow L^{2}(\Omega)_{u}$ be the orthogonal projection from $H$ onto the first component space associated with the u-component. Let $B_{0}$ be the bounded absorbing set of $\{S(t)\}_{t \geq 0}$ in $H$, shown in Lemma 3. Then for any given $M>0$, it holds that

$$
\kappa\left(P_{u}\left(S(t) B_{0}\right)_{\Omega(|v(t)|<M)}\right) \longrightarrow 0, \text { as } t \rightarrow \infty
$$


where $\left(S(t) B_{0}\right)_{\Omega(|v(t)|<M)}$ has been specified in Theorem 3.

Proof. We still use the notation $\Omega_{v, M}=\Omega(|v(t)|<M)$ as in Lemma 7. Taking the inner-product $\langle(1.1),-\Delta u(t)\rangle_{\Omega_{v, M}}$, we can get

$$
\begin{aligned}
& \frac{1}{2} \frac{d}{d t}\|\nabla u\|_{\Omega_{v, M}}^{2}+d_{1}\|\Delta u\|_{\Omega_{v, M}}^{2}=\int_{\Omega_{v, M}} u^{2} v(-\Delta u) d x \\
& \quad+(b+1) \int_{\Omega_{v, M}} u \Delta u d x-\int_{\Omega_{v, M}} a \Delta u d x \\
& \quad \leq M \int_{\Omega_{v, M}} u^{2}|\Delta u| d x+\frac{d_{1}}{4}\|\Delta u\|_{\Omega_{v, M}}^{2}+\frac{1}{d_{1}}\|(b+1) u-a\|_{\Omega_{v, M}}^{2} \\
& \quad \leq \frac{M^{2}}{d_{1}} \int_{\Omega_{v, M}} u^{4} d x+\frac{d_{1}}{2}\|\Delta u\|_{\Omega_{v, M}}^{2}+\frac{1}{d_{1}}\|(b+1) u-a\|_{\Omega_{v, M}}^{2} .
\end{aligned}
$$

Note that for $n \leq 3$ the Sobolev embedding $H_{0}^{1}(\Omega) \hookrightarrow L^{4}(\Omega)$ is continuous and there exists a uniform constant $\delta>0$ such that

$$
\|\varphi\|_{L^{4}(\Omega)}^{2} \leq \delta\|\varphi\|_{H_{0}^{1}(\Omega)}^{2}, \quad \text { for } \varphi \in H_{0}^{1}(\Omega),
$$

which holds also for functions on $\Omega_{v, M}$. Thus it follows that

$$
\begin{aligned}
& \frac{d}{d t}\|\nabla u\|_{\Omega_{v, M}}^{2}+d_{1} \gamma\|\nabla u\|_{\Omega_{v, M}}^{2} \\
& \leq \frac{2 M^{2}}{d_{1}}\|u\|_{L^{4}\left(\Omega_{v, M}\right)}^{4}+\frac{4}{d_{1}}\left((b+1)^{2} K_{0}+a^{2}\left|\Omega_{v, M}\right|\right) \\
& \quad \leq \frac{2 M^{2} \delta^{2}}{d_{1}}\|\nabla u\|^{4}+\frac{4}{d_{1}}\left((b+1)^{2} K_{0}+a^{2}\left|\Omega_{v, M}\right|\right)
\end{aligned}
$$

for $t \geq T_{0}$, where $T_{0}$ is given at the beginning of the proof of Lemma 6 . The inequality (4.24) implies that

$$
\frac{d y}{d t} \leq g y+h, \quad \text { for } t \geq T_{0},
$$

where

$$
\begin{aligned}
& y(t)=\|\nabla u\|_{\Omega_{v, M}}^{2}, \quad g(t)=\frac{2 M^{2} \delta^{2}}{d_{1}}\|\nabla u\|_{\Omega_{v, M}}^{2}, \quad \text { and } \\
& h(t)=\frac{4}{d_{1}}\left((b+1)^{2} K_{0}+a^{2}\left|\Omega_{v, M}\right|\right) .
\end{aligned}
$$

In view of (4.13), we can apply the uniform Gronwall inequality to (4.25) to obtain

$$
\begin{aligned}
& \|\nabla u(t)\|_{\Omega_{v, M}}^{2} \leq \\
& {\left[C_{3}(M)+\frac{4}{d_{1}}\left((b+1)^{2} K_{0}+a^{2}\left|\Omega_{v, M}\right|\right)\right] \exp \left(\frac{2 M^{2} \delta^{2}}{d_{1}} C_{3}(M)\right),} \\
& \quad \text { for } t \geq T_{4}=\max \left\{T_{0}\left(B_{0}\right), T_{3}\left(B_{0}\right)\right\}+1=T_{3}\left(B_{0}\right)+1,
\end{aligned}
$$

where $T_{3}$ is given in the proof of Lemma 7 . Inequality (4.26) shows that

$$
P_{u}\left(S(t) B_{0}\right)_{\Omega(|v(t)|<M)} \text { is a bounded set in } H_{0}^{1}(\Omega) \text {, for any } t \geq T_{4} \text {, }
$$


so that

$$
P_{u}\left(S(t) B_{0}\right)_{\Omega(|v(t)|<M)} \text { is a precompact set in } L^{2}(\Omega) .
$$

Therefore, by Lemma 4 , it is proved that in $L^{2}(\Omega)$,

$$
\kappa\left(P_{u}\left(S(t) B_{0}\right)_{\Omega(|v(t)|<M)}\right)=0, \quad \text { for } t \geq T_{4},
$$

and (4.22) is valid. The proof is completed.

Assembling the results shown in Lemma 6 through Lemma 9, we have proved that the Brusselator semiflow $\{S(t)\}_{t \geq 0}$ has the $\kappa$-contracting property. We now give the proof of Theorem 1 (Main Theorem) as follows.

Proof. By Lemma $3,\{S(t)\}_{t \geq 0}$ has a bounded absorbing set $B_{0}$ in $H$ and the condition (i) in Theorem 3 is satisfied. According to Lemmas 6 through 9, this $\{S(t)\}_{t \geq 0}$ satisfies the conditions (ii) in Theorem 3. Then by Theorem 3, there exists a global attractor $\mathcal{A}$ in $H$ for the Brusselator semiflow $\{S(t)\}_{t \geq 0}$.

By the same approach, we can prove the existence of a global attractor for all the Gray-Scott equations, the Glycolysis equations, and the Schnackenberg equations mentioned in Section 1 as well. For the Gray-Scott equations,

$$
\begin{aligned}
& \frac{\partial u}{\partial t}=d_{1} \Delta u-(F+k) u+u^{2} v, \\
& \frac{\partial v}{\partial t}=d_{2} \Delta v+F(1-v)-u^{2} v,
\end{aligned}
$$

we can prove the following result.

TheOREM 4. For any positive parameters $d_{1}, d_{2}, F$ and $k$, there exists a global attractor $\mathcal{A}_{\mathcal{Q}}$ in $H$ for the solution semiflow $\{Q(t)\}_{t \geq 0}$ generated by the Gray-Scott equations (4.27)-(4.28).

Proof. Very similar to (2.1), we have the following inequality for the $v$ component,

$$
\begin{aligned}
\frac{1}{2} \frac{d}{d t}\|v\|^{2}+d_{2}\|\nabla v\|^{2} & =\int_{\Omega}\left(-u^{2} v^{2}-F v^{2}\right) d x+\int_{\Omega} F v d x \\
& \leq \frac{1}{2}\left(d_{2} \gamma\|v\|^{2}+\frac{F^{2}}{d_{2} \gamma}|\Omega|\right)
\end{aligned}
$$

so that

$$
\frac{d}{d t}\|v\|^{2}+d_{2} \gamma\|v\|^{2} \leq \frac{F^{2}}{d_{2} \gamma}|\Omega| .
$$

On the other hand, for the Gray-Scott equations, $z(t)=u(t)+v(t)$ satisfies the following equation which is very similar to (2.5) in the Brusselator case,

$$
z_{t}=d_{1} \Delta z-(F+k) z+\left[\left(d_{2}-d_{1}\right) \Delta v+k v+F\right] .
$$

Starting from (4.29) and (4.31), and exactly parallel to the lemmas shown in Section 2 and Section 4, one can prove the existence of a global attractor in $H$ for the solution semiflow $\{Q(t)\}_{t \geq 0}$ generated by the Gray-Scott equations (4.27)-(4.28). We omit the details. 
For the Glycolysis equations,

$$
\begin{aligned}
& \frac{\partial u}{\partial t}=d_{1} \Delta u-u+k v+u^{2} v+\rho, \\
& \frac{\partial v}{\partial t}=d_{2} \Delta v-k v-u^{2} v+\delta,
\end{aligned}
$$

we can also prove the existence of a global attractor in the same way.

THEOREM 5. For any positive parameters $d_{1}, d_{2}, k, \rho$, and $\delta$, there exists a global attractor $\mathcal{A}_{\mathcal{G}}$ in $H$ for the solution semiflow $\{G(t)\}_{t>0}$ generated by the Glycolysis equations (4.32)-(4.33).

Proof. Correspondingly we have the following inequality for the $v$-component,

$$
\begin{aligned}
\frac{1}{2} \frac{d}{d t}\|v\|^{2}+d_{2}\|\nabla v\|^{2} & =\int_{\Omega}\left(-u^{2} v^{2}-k v^{2}\right) d x+\int_{\Omega} \delta v d x \\
& \leq \frac{1}{2}\left(d_{2} \gamma\|v\|^{2}+\frac{\delta^{2}}{d_{2} \gamma}|\Omega|\right),
\end{aligned}
$$

and the following equation satisfied by $z(t)=u(t)+v(t)$,

$$
z_{t}=d_{1} \Delta z-z+\left[\left(d_{2}-d_{1}\right) \Delta v+v+(\rho+\delta)\right] .
$$

Starting from (4.34) and (4.35), exactly parallel to the lemmas shown in Section 2 and Section 4, one can prove the existence of a global attractor in $H$ for the solution semiflow $\{G(t)\}_{t \geq 0}$ generated by the Glycolysis equations (4.32)-(4.33).

For the Schnackenberg equations $[\mathbf{2 3}, \mathbf{3 1}]$,

$$
\begin{aligned}
& \frac{\partial u}{\partial t}=d_{1} \Delta u-k u+u^{2} v+a, \\
& \frac{\partial v}{\partial t}=d_{2} \Delta v-u^{2} v+b
\end{aligned}
$$

the following theorem also holds.

TheOREM 6. For any positive parameters $d_{1}, d_{2}, k, a$, and $b$, there exists a global attractor $\mathcal{A}_{\mathcal{R}}$ in $H$ for the solution semiflow $\{R(t)\}_{t \geq 0}$ generated by the Schnackenberg equations (4.36)-(4.37).

Proof. In this case, we have the following inequality for the $v$-component,

$$
\frac{1}{2} \frac{d}{d t}\|v\|^{2}+d_{2}\|\nabla v\|^{2}=\int_{\Omega}\left(-u^{2} v^{2}+b v\right) d x \leq \frac{1}{2}\left(d_{2} \gamma\|v\|^{2}+\frac{b^{2}}{d_{2} \gamma}|\Omega|\right),
$$

and the following equation satisfied by $z(t)=u(t)+v(t)$,

$$
z_{t}=d_{1} \Delta z-k z+\left[\left(d_{2}-d_{1}\right) \Delta v+k v+(a+b)\right] .
$$

Therefore, starting from (4.38) and (4.39), and exactly parallel to the lemmas shown in Section 2 and Section 4, one can prove the existence of a global attractor in $H$ for the solution semiflow $\{R(t)\}_{t \geq 0}$ generated by the Schnackenberg equations (4.36)(4.37). 


\section{Finite Dimensionality of Global Attractor}

In this section we shall prove that the global attractor $\mathcal{A}$ for the Brusselator semiflow has finite Hausdorff and fractal dimensions and estimate upper bounds for these dimensions.

Definition 6. Let $X$ be a complete metric space and $Y$ be a subset of $X$. Given $d>0$, the $d$-dimensional Hausdorff measure of $Y$ is defined by

where

$$
\mu_{H}(Y, d)=\lim _{\varepsilon \rightarrow 0^{+}} \mu(Y, d, \varepsilon)=\sup _{\varepsilon>0} \mu(Y, d, \varepsilon)
$$

$$
\mu(Y, d, \varepsilon)=\inf \sum_{i \in I} r_{i}^{d}
$$

where the infimum is taken over all coverings of $Y$ by a family of balls $\left\{B_{i}\right\}_{i \in I}$ in $X$ of radii $r_{i} \leq \varepsilon$. The Hausdorff dimension of $Y$ is defined as the unique number $d_{H}(Y) \in[0, \infty]$ which satisfies the folllowing two conditions,

$$
\begin{aligned}
& \mu_{H}(Y, d)=0, \quad \text { for } d>d_{H}(Y), \\
& \mu_{H}(Y, d)=\infty, \text { for } d<d_{H}(Y) .
\end{aligned}
$$

Definition 7. The fractal dimension of a subset $Y$ in a complete metric space $X$ is defined as the number

$$
d_{F}(Y)=\limsup _{\varepsilon \rightarrow 0^{+}} \frac{\log n_{Y}(\varepsilon)}{\log 1 / \varepsilon},
$$

where $n_{Y}(\varepsilon)$ is the minimum number of balls in $X$ of radius $\varepsilon$ which cover the set $Y$.

One can use the alternative expression $[\mathbf{2 9}]$ for $d_{F}(Y)$ :

$$
d_{F}(Y)=\inf \left\{d>0: \mu_{F}(Y, d)=\limsup _{\varepsilon \rightarrow 0^{+}}\left(\varepsilon^{d} n_{Y}(\varepsilon)\right)=0\right\} .
$$

Note that $\mu_{H}(Y, d) \leq \mu_{F}(Y, d)$ and $d_{H}(Y) \leq d_{F}(Y)$.

The following lemma [29, Chapter 5] based on the theory of Lyapunov exponents will be used to estimate the upper bounds of the Hausdorff and fractal dimensions of the global attractor $\mathcal{A}$.

Lemma 10. Let $\mathcal{A}$ be the global attractor of the Brusselator semiflow $\{S(t)\}_{t \geq 0}$ in H. Define

$$
\begin{gathered}
q_{m}(t)=\sup _{w_{0} \in \mathcal{A}} \sup _{\substack{\xi_{i} \in H,\left\|\xi_{i}\right\|=1 \\
i=1, \cdots, m}}\left(\frac{1}{t} \int_{0}^{t} \operatorname{Tr}\left(A+F^{\prime}\left(S(\tau) w_{0}\right)\right) \circ Q_{m}(\tau) d \tau\right), \\
q_{m}=\limsup _{t \rightarrow \infty} q_{m}(t),
\end{gathered}
$$

where $\operatorname{Tr}\left(A+F^{\prime}\left(S(\tau) w_{0}\right)\right)$ is the trace of the linear operator $A+F^{\prime}\left(S(\tau) w_{0}\right)$, with $F(w)$ being the nonlinear map in (1.6), and $Q_{m}(t)$ stands for the orthogonal projection of the space $H$ on the subspace spanned by $y_{1}(t), \cdots, y_{m}(t)$, with

$$
y_{i}(t)=L\left(S(t) w_{0}\right) \xi_{i}, \quad i=1, \cdots, m .
$$

Here $F^{\prime}\left(S(\tau) w_{0}\right)$ is the Fréchet derivative of that map $F$ at $S(\tau) w_{0}$, and $L\left(S(t) w_{0}\right)$ is the Fréchet derivative of the map $S(t)$ at $w_{0}$, with $t$ being fixed. If there is an 
integer $m$ such that $q_{m}<0$, then the Hausdorff dimension and the fractal dimension of $\mathcal{A}$ satisfy, respectively,

$$
\begin{aligned}
& d_{H}(\mathcal{A}) \leq m, \text { and } \\
& d_{F}(\mathcal{A}) \leq m \max _{1 \leq j \leq m-1}\left(1+\frac{\left(q_{j}\right)_{+}}{\left|q_{m}\right|}\right) \leq 2 m .
\end{aligned}
$$

First it can be shown that for any given $t>0, S(t)$ is Fréchet differentiable in $H$ and its Fréchet derivative at $w_{0}$ is the bounded linear operator $L\left(S(t) w_{0}\right)$ given by

$$
L\left(S(t) w_{0}\right) \xi \stackrel{\text { def }}{=} y(t)=(U(t), V(t)), \quad \text { for any } \xi=(\eta, \zeta) \in H,
$$

where $(U(t), V(t))$ is the strong solution of the following Brusselator variational equation

$$
\begin{gathered}
\frac{\partial U}{\partial t}=d_{1} \Delta U+2 u(t) v(t) U+u^{2}(t) V-(b+1) U \\
\frac{\partial V}{\partial t}=d_{2} \Delta V-2 u(t) v(t) U-u^{2}(t) V+b U \\
U(0)=\eta, \quad V(0)=\zeta .
\end{gathered}
$$

Here $w(t)=(u(t), v(t))=S(t) w_{0}$ is the solution of the Brusselator evolutionary equation (1.6) with the initial condition $w(0)=w_{0}$. Due to the space limitation, we omit the detailed verification of this fact. The initial value problem (5.6)-(5.7)-(5.8) can be written as

$$
\frac{d y}{d t}=\left(A+F^{\prime}\left(S(t) w_{0}\right)\right) y, \quad y(0)=\xi .
$$

Note that the invariance of $\mathcal{A}$ implies $\mathcal{A} \subset B_{0}$, where $B_{0}$ is the bounded absorbing set given in Lemma 3. Hence we have

$$
\sup _{w_{0} \in \mathcal{A}}\left\|S(t) w_{0}\right\|^{2} \leq K_{0}
$$

where $K_{0}$ is the constant given in (2.17). Here we need one more property of the global attractor $\mathcal{A}$, stated in the next lemma.

Lemma 11. Let $\mathcal{A}$ be the global attractor of the Brusselator semiflow $\{S(t)\}_{t \geq 0}$ in $H$. There there exists a uniform constant $K_{1}>0$ such that

$$
\|\nabla w\|^{2} \leq K_{1}, \quad \text { for any } w \in \mathcal{A} .
$$

Proof. In (1.6), $A: D(A)(=W) \rightarrow H$ is a positive sectorial operator and $F \in C_{\mathrm{loc}}^{\operatorname{Lip}}(E, H)$. By (1.5), for any $w_{0} \in \mathcal{A}$, there is a $t_{0} \in\left(0, \frac{1}{2}\right)$ such that $S\left(t_{0}\right) w_{0} \in E$. Since $\mathcal{A} \subset H$ is compact and invariant, $S(t) \mathcal{A}=\mathcal{A}$, by the solution theory in $[\mathbf{2 4}$, Section 4.7], one has

$$
S(\cdot) w_{0} \in C\left(\left[t_{0}, \infty\right), E\right) \cap C_{\mathrm{loc}}^{0, \frac{1}{2}}\left(\left(t_{0}, \infty\right), E\right) \cap C\left(\left(t_{0}, \infty\right), W\right),
$$

were $C_{\text {loc }}^{0, \frac{1}{2}}$ stands for the space of Hölder strongly continuous functions with exponent $1 / 2$. Since for any $\widehat{w} \in \mathcal{A}$ and any $t \geq 1$, there is a particular $w_{0} \in \mathcal{A}$ such 
that $\widehat{w}=S(t) w_{0}$, the global attractor has the regularity $\mathcal{A} \subset E$. But this does not imply in general that $\mathcal{A}$ attracts bounded sets with respect to the norm of $E$.

Next we prove that $\mathcal{A}$ is a bounded set in $E$. Suppose the contrary. Then there exist sequences $\left\{N_{\ell}\right\} \subset(0, \infty)$, with $N_{\ell} \geq \ell$, and $\left\{w_{\ell}\right\} \subset \mathcal{A}$, such that

$$
\left\|\nabla w_{\ell}\right\| \geq N_{\ell}, \quad \ell=1,2, \cdots .
$$

Let $w_{\ell}^{0} \in \mathcal{A}$ be given such that $w_{\ell}=S\left(N_{\ell}\right) w_{\ell}^{0}$. By (5.11), there is a Hölder constant $c_{0}>0$ such that

$$
\left\|\nabla S(t) w_{\ell}^{0}\right\|^{2} \geq\left(N_{\ell}-\frac{c_{0}}{\sqrt{2}}\right)_{+}^{2}, \quad \text { for } t \in I_{\ell}=\left(N_{\ell}-\frac{1}{2}, N_{\ell}+\frac{1}{2}\right) .
$$

This shows that

$$
\int_{N_{\ell}-\frac{1}{2}}^{N_{\ell}+\frac{1}{2}}\left\|\nabla S(\tau) w_{\ell}^{0}\right\|^{2} d \tau \geq\left(N_{\ell}-\frac{c_{0}}{\sqrt{2}}\right)_{+}^{2} \longrightarrow \infty, \quad \text { as } \ell \rightarrow \infty .
$$

This is a contradiction to the fact that for any $w_{0} \in \mathcal{A}$, due to $(2.4)$,

$$
\int_{t}^{t+1}\|\nabla v(s)\|^{2} d s \leq c_{1}=\frac{1}{d_{2}}\left(K_{0}+\frac{b^{2}|\Omega|}{2 \gamma d_{2}}\right)+\frac{b^{2}|\Omega|}{d_{2}}, \quad \text { for any } t>0,
$$

and, due to (2.6),

$$
\int_{t}^{t+1}\|\nabla z(s)\|^{2} d s \leq c_{2}=\frac{1}{d_{1}}\left[3 K_{0}+\frac{\left|d_{2}-d_{1}\right|^{2}}{d_{1}} c_{1}+\left(\frac{b^{2}}{\gamma d_{2}}+2 a^{2}\right)|\Omega|\right],
$$

for any $t>0$, where $c_{1}$ and $c_{2}$ are uniform constants. Therefore, the conclusion is valid.

Now we can prove the finite dimensionality of the global attractors.

Theorem 7. The global attractors $\mathcal{A}$ for the Brusselator equations, $\mathcal{A}_{\mathcal{Q}}$ for the Gray-Scott equations, $\mathcal{A}_{\mathcal{G}}$ for the Glycolysis equations, and $\mathcal{A}_{\mathcal{R}}$ for the Schnackenberg equations all have finite Hausdorff dimenions and fractal dimensions, respectively.

Proof. By Lemma 10, we shall estimate $\operatorname{Tr}\left(A+F^{\prime}\left(S(\tau) w_{0}\right)\right) \circ Q_{m}(\tau)$. At any given time $\tau>0$, let $\left\{\varphi_{j}(\tau): j=1, \cdots, m\right\}$ be an $H$-orthonormal basis for the subspace

$$
Q_{m}(\tau) H=\operatorname{Span}\left\{y_{1}(\tau), \cdots, y,(\tau)\right\}
$$

where $y(t)=\left(y_{1}(t), \cdots, y_{m}(t)\right)$ satisfies (5.9) with $y(0)=\xi=\left(\xi_{1}, \cdots, \xi_{m}\right)$, and without loss of generality assuming that $\left\{\xi_{1}, \cdots, \xi_{m}\right\}$ is a linearly independent set in $H$. By the Gram-Schmidt orthogonalization scheme, $\varphi_{j}(\tau)=\left(\varphi_{j}^{1}(\tau), \varphi_{j}^{2}(\tau)\right) \in E$, for $j=1, \cdots, m$, since $y_{1}(\tau), \cdots, y_{m}(\tau) \in E$, for $\tau>0$, and $\varphi_{j}(\tau)$ are strongly measurable in $\tau$. Let $d_{0}=\min \left\{d_{1}, d_{2}\right\}$. Then we have

$$
\begin{aligned}
\operatorname{Tr} & \left(A+F^{\prime}\left(S(\tau) w_{0}\right) \circ Q_{m}(\tau)\right. \\
& =\sum_{j=1}^{m}\left\langle A \varphi_{j}(\tau), \varphi_{j}(\tau)\right\rangle+\sum_{j=1}^{m}\left\langle F^{\prime}\left(S(\tau) w_{0}\right) \varphi_{j}(\tau), \varphi_{j}(\tau)\right\rangle \\
& \leq-d_{0} \sum_{j=1}^{m}\left\|\nabla \varphi_{j}(\tau)\right\|^{2}+J_{1}+J_{2}+J_{3},
\end{aligned}
$$


where

$$
\begin{gathered}
J_{1}=\sum_{j=1}^{m} \int_{\Omega} 2 u(\tau) v(\tau)\left(\left|\varphi_{j}^{1}(\tau)\right|^{2}-\varphi_{j}^{1}(\tau) \varphi_{j}^{2}(\tau)\right) d x \\
\leq \sum_{j=1}^{m} \int_{\Omega} 2|u(\tau)||v(\tau)|\left(\left|\varphi_{j}^{1}(\tau)\right|^{2}+\left|\varphi_{j}^{1}(\tau)\right|\left|\varphi_{j}^{2}(\tau)\right|\right) d x \\
J_{2}=\sum_{j=1}^{m} \int_{\Omega} u^{2}(\tau)\left(\varphi_{j}^{1}(\tau) \varphi_{j}^{2}(\tau)-\left|\varphi_{j}^{2}(\tau)\right|^{2}\right) d x \leq \sum_{j=1}^{m} \int_{\Omega} u^{2}(\tau)\left|\varphi_{j}^{1}(\tau)\right|\left|\varphi_{j}^{2}(\tau)\right| d x
\end{gathered}
$$

and

$$
\begin{aligned}
J_{3} & =\sum_{j=1}^{m} \int_{\Omega}\left(-(b+1)\left|\varphi_{j}^{1}(\tau)\right|^{2}+b \varphi_{j}^{1}(\tau) \varphi_{j}^{2}(\tau)\right) d x \\
& \leq \sum_{j=1}^{m} \int_{\Omega} b \varphi_{j}^{1}(\tau) \varphi_{j}^{2}(\tau) d x .
\end{aligned}
$$

We can estimate each of the three terms as follows. First, by the generalized Hölder inequality and the Sobolev embedding $E \hookrightarrow L^{4}(\Omega) \times L^{4}(\Omega)$ for $n \leq 3$, and using Lemma 11, we get

$$
\begin{aligned}
J_{1} & \leq 2 \sum_{j=1}^{m}\|u(\tau)\|_{L^{4}}\|v(\tau)\|_{L^{4}}\left(\left\|\varphi_{j}^{1}(\tau)\right\|_{L^{4}}^{2}+\left\|\varphi_{j}^{1}(\tau)\right\|_{L^{4}}\left\|\varphi_{j}^{2}(\tau)\right\|_{L^{4}}\right) \\
& \leq 4 \sum_{j=1}^{m}\left\|S(\tau) w_{0}\right\|_{L^{4}}^{2}\left\|\varphi_{j}(\tau)\right\|_{L^{4}}^{2} \leq 4 \delta \sum_{j=1}^{m}\left\|\nabla S(\tau) w_{0}\right\|^{2}\left\|\varphi_{j}(\tau)\right\|_{L^{4}}^{2} \\
& \leq 4 \delta K_{1} \sum_{j=1}^{m}\left\|\varphi_{j}(\tau)\right\|_{L^{4}}^{2}
\end{aligned}
$$

where $\delta$ is the Sobolev embedding coefficient given in (4.23). Now we apply the Garliardo-Nirenberg interpolation inequality, cf. [24, Theorem B.3],

$$
\|\varphi\|_{W^{k, p}} \leq C\|\varphi\|_{W^{m, q}}^{\theta}\|\varphi\|_{L^{r}}^{1-\theta}, \quad \text { for } \varphi \in W^{m, q}(\Omega),
$$

provided that $p, q, r \geq 1,0<\theta \leq 1$, and

$$
k-\frac{n}{p} \leq \theta\left(m-\frac{n}{q}\right)-(1-\theta) \frac{n}{r}, \quad \text { where } n=\operatorname{dim} \Omega .
$$

Here with $W^{k, p}(\Omega)=L^{4}(\Omega), W^{m, q}(\Omega)=H_{0}^{1}(\Omega), L^{r}(\Omega)=L^{2}(\Omega)$, and $\theta=n / 4 \leq$ $3 / 4$, it follows from $(5.14)$ that

$$
\left\|\varphi_{j}(\tau)\right\|_{L^{4}} \leq C\left\|\nabla \varphi_{j}(\tau)\right\|^{\frac{n}{4}}\left\|\varphi_{j}(\tau)\right\|^{1-\frac{n}{4}}=C\left\|\nabla \varphi_{j}(\tau)\right\|^{\frac{n}{4}}, \quad j=1, \cdots, m
$$

since $\left\|\varphi_{j}(\tau)\right\|=1$, where $C$ is a uniform constant. Substitute (5.15) into (5.13) to obtain

$$
J_{1} \leq 4 \delta K_{1} C^{2} \sum_{j=1}^{m}\left\|\nabla \varphi_{j}(\tau)\right\|^{\frac{n}{2}}
$$


Similarly, by the generalized Hölder inequality, we can get

$$
J_{2} \leq \delta K_{1} \sum_{j=1}^{m}\left\|\varphi_{j}(\tau)\right\|_{L^{4}}^{2} \leq \delta K_{1} C^{2} \sum_{j=1}^{m}\left\|\nabla \varphi_{j}(\tau)\right\|^{\frac{n}{2}} .
$$

Moreover, we have

$$
J_{3} \leq \sum_{j=1}^{m} b\left\|\varphi_{j}(\tau)\right\|^{2}=b m .
$$

Substituting (5.16), (5.17) and (5.18) into (5.12), we obtain

$$
\begin{aligned}
& \operatorname{Tr}\left(A+F^{\prime}\left(S(\tau) w_{0}\right) \circ Q_{m}(\tau) \leq-d_{0} \sum_{j=1}^{m}\left\|\nabla \varphi_{j}(\tau)\right\|^{2}+\right. \\
& 5 \delta K_{1} C^{2} \sum_{j=1}^{m}\left\|\nabla \varphi_{j}(\tau)\right\|^{\frac{n}{2}}+b m .
\end{aligned}
$$

By Young's inequality, for $n \leq 3$, we have

$$
5 \delta K_{1} C^{2} \sum_{j=1}^{m}\left\|\nabla \varphi_{j}(\tau)\right\|^{\frac{n}{2}} \leq \frac{d_{0}}{2} \sum_{j=1}^{m}\left\|\nabla \varphi_{j}(\tau)\right\|^{2}+K_{2}(n) m,
$$

where $K_{2}(n)$ is a uniform constant depending only on $n=\operatorname{dim}(\Omega)$. Hence,

$$
\operatorname{Tr}\left(A+F^{\prime}\left(S(\tau) w_{0}\right) \circ Q_{m}(\tau) \leq-\frac{d_{0}}{2} \sum_{j=1}^{m}\left\|\nabla \varphi_{j}(\tau)\right\|^{2}+\left(K_{2}(n)+b\right) m .\right.
$$

According to the generalized Sobolev-Lieb-Thirring inequality [29, Appendix, Corollary 4.1], since $\left\{\varphi_{1}(\tau), \cdots, \varphi_{m}(\tau)\right\}$ is an orthonormal set in $H$, so there exists a uniform constant $K_{3}>0$ only depending on the shape and dimension of $\Omega$, such that

$$
\sum_{j=1}^{m}\left\|\nabla \varphi_{j}(\tau)\right\|^{2} \geq K_{3} \frac{m^{1+\frac{2}{n}}}{|\Omega|^{\frac{2}{n}}}
$$

Therefore, we end up with

$$
\operatorname{Tr}\left(A+F^{\prime}\left(S(\tau) w_{0}\right) \circ Q_{m}(\tau) \leq-\frac{d_{0} K_{3}}{2|\Omega|^{\frac{2}{n}}} m^{1+\frac{2}{n}}+\left(K_{2}(n)+b\right) m .\right.
$$

Then we can conclude that

$$
\begin{aligned}
q_{m}(t) & =\sup _{w_{0} \in \mathcal{A}} \sup _{\substack{\xi_{i} \in H,\left\|\xi_{i}\right\|=1 \\
i=1, \cdots, m}}\left(\frac{1}{t} \int_{0}^{t} \operatorname{Tr}\left(A+F^{\prime}\left(S(\tau) w_{0}\right)\right) \circ Q_{m}(\tau) d \tau\right) \\
& \leq-\frac{d_{0} K_{3}}{2|\Omega| \frac{2}{n}} m^{1+\frac{2}{n}}+\left(K_{2}(n)+b\right) m, \quad \text { for any } t>0,
\end{aligned}
$$

so that

$$
q_{m}=\limsup _{t \rightarrow \infty} q_{m}(t) \leq-\frac{d_{0} K_{3}}{2|\Omega|^{\frac{2}{n}}} m^{1+\frac{2}{n}}+\left(K_{2}(n)+b\right) m<0,
$$

if the integer $m$ satisfies the following condition,

$$
m-1 \leq\left(\frac{2\left(K_{2}(n)+b\right)}{d_{0} K_{3}}\right)^{n / 2}|\Omega|<m .
$$


According to Lemma 10, we have shown that the Hausdorff dimension and the fractal dimension of the global attractor $\mathcal{A}$ are finite and their upper bounds are given by

$$
d_{H}(\mathcal{A}) \leq m \quad d_{F}(\mathcal{A}) \leq 2 m
$$

where $m$ satisfies (5.24). Thus the theorem is proved for the Brusselator semiflow. The proof for the other three model equations is done similarly.

\section{References}

[1] M. Al-Ghoul and B.C. Eu, Hyperbolic reaction-diffusion equations, patterns, and phase speeds for the Brusselator, J. Phys. Chemistry, 100 (1996), 18900-18910.

[2] M. Ashkenazi and H.G. Othmer, Spatial patterns in coupled biochemical oscillators, J. Math. Biology, 5 (1978), 305-350.

[3] J.F.G. Auchmuty and G. Nicolis, Bifurcation analysis of nonlinear reaction-diffusion equations - I: Evolution equations and the steady state solutions, Bull. Math. Biology, 37 (1975), 323-365.

[4] P.W. Bates, K. Lu, and B. Wang, Attractors for lattice dynamical systems, Intern. J. Bifurcation and Chaos, 11 (2001), 143-153.

[5] K.J. Brown and F.A. Davidson, Global bifurcation in the Brusselator system, Nonlinear Analysis, 24 (1995), 1713-1725.

[6] A. Doelman, T.J. Kaper, and P.A. Zegeling, Pattern formation in the one-dimensional GrayScott model, Nonlinearity, 10 (1997), 523-563.

[7] I.R. Epstein, Complex dynamical behavior in simple chemical systems, J. Phys. Chemistry, 88 (1984), 187-198.

[8] I.R. Epstein and J.A. Pojman, An Introduction to Nonlinear Chemical Dynamics, Oxford Univ. Press, New York, 1998.

[9] T. Erneux and E. Reiss, Brusselator isolas, SIAM J. Appl. Math., 43 (1983), 1240-1246.

[10] R.J. Fields and M. Burger, Oscillation and Traveling Waves in Chemical Systems, John Wiley and Sons, 1985.

[11] P. Gray and S.K. Scott, Sustained oscillations and other exotic patterns of behavior in isothermal reactions, J. Phys. Chemistry, 89 (1985), 22-32.

[12] P. Gray and S.K. Scott, Chemical Waves and Instabilities, Clarendon, Oxford, 1990.

[13] J.K. Hale, Asymptotic Behavior of Dissipative Systems, Amer. Math. Soc., Providence, RI, 1988.

[14] T. Kolokolnikov, T. Erneux, and J. Wei, Mesa-type patterns in one-dimensional Brusselator and their stability, Physica D, 214(1) (2006), 63-77.

[15] K.J. Lee, W.D. McCormick, Q. Ouyang, and H. Swinney, Pattern formation by interacting chemical fronts, Science, 261 (1993), 192-194.

[16] R. Lefever and G. Nicolis, Chemical instabilities and sustained oscillations, J. Theoretical Biology, 30 (1971), 267-284.

[17] Q. Ma, S. Wang, and C. Zhong, Necessary and sufficient conditions for the existence of global attractors for semigroups and applications, Indiana Univ. Math. Journal, 51 (2002), 1541-1559.

[18] J.E. Pearson, Complex patterns in a simple system, Science, 261 (1993), 189-192.

[19] I. Prigogine and R. Lefever, Symmetry-breaking instabilities in dissipative systems, J. Chem. Physics, 48 (1968), 1665-1700.

[20] B. Peña and C. Pérez-García, Stability of Turing patterns in the Brusselator model, Phys. Review E, 64(5), 2001.

[21] Ya.B. Pesin and A.A. Yurchenko, Some physical models of the reaction-diffusion equation and coupled map lattices, Russian Math. Surveys, 59(3) (2004), 481-513.

[22] T. Rauber and G. Runger, Aspects of a distributed solution of the Brusselator equation, Proc. of the First Aizu International Symposium on Parallel Algorithms and Architecture Syntheses, (1995), 114-120.

[23] J. Schnackenberg, Simple chemical reaction systems with limit cycle behavior, J. Theor. Biology, 81 (1979), 389-400.

[24] G.R. Sell and Y. You, Dynamics of Evolutionary Equations, Springer, New York, 2002. 
[25] M. Stanislavova, A. Stefanov, and B. Wang, Asymptotic smoothing and attractors for the generalized Benjamin-Bona-Mahony equations on $\Re^{3}$, J. Dff. Eqns., 219 (2005), 451-483.

[26] S.H. Strogatz, Nonlinear Dynamics and Chaos, Westview Press, 1994.

[27] C. Sun, M. Yang, and C. Zhong, Global attractors for the wave equation with nonlinear damping, J. Diff. Eqns., 227 (2006), 427-443.

[28] C. Sun and C. Zhong, Attractors for the semilinear reaction-diffusion equation with distributed derivatives in unbounded domains, Nonlinear Analysis, 63 (2005), 49-65.

[29] R. Temam, Infinite Dimensional Dynamical Systems in Mechanics and Physics, SpringerVerlag, New York, 1988.

[30] B. Wang, Attractors for reaction-diffusion equation in unbounded domains, Physica D, 128 (1999), 41-52.

[31] M.J. Ward and J. Wei, The existence and stability of asymmetric spike patterns for the Schnackenberg model, Stud. Appl. Math., 109 (2002), 229-264.

[32] J. Wei and M. Winter, Asymmetric spotty patterns for the Gray-Scott model in $\Re^{2}$, Stud. Appl. math., 110 (2003), 63-102.

[33] A. De Wit, D. Lima, G. Dewel, and P. Borckmans, Spatiotemporal dynamics near a codimension-two point, Phys. Review E, 54 (1), 1996.

[34] Y. You, Global dynamics of nonlinear wave equations with cubic non-monotone damping, Dynamics of PDE, 1 (2004), 65-86.

[35] Y. You, Finite dimensional reduction of global dynamics and lattice dynamics of a damped nonlinear wave equation, in "Control Theory and mathematical Finance", edit. S. Tang and J. Yong, World Scientific, 2005, in press.

Department of Mathematics and Statistics, University of South Florida, Tampa, FL 33620

E-mail address: you@math.usf.edu 